\title{
A Parametric Sharpe Ratio Optimization Approach for Fuzzy Portfolio Selection Problem
}

\author{
Ying Liu and Ya-Nan Li \\ Risk Management \& Financial Engineering Laboratory, College of Mathematics and Information Science, \\ Hebei University, Baoding, Hebei 071002, China
}

Correspondence should be addressed to Ya-Nan Li; yananli071002@sina.com

Received 15 August 2016; Revised 11 December 2016; Accepted 14 December 2016; Published 31 January 2017

Academic Editor: Shuming Wang

Copyright (C) 2017 Ying Liu and Ya-Nan Li. This is an open access article distributed under the Creative Commons Attribution License, which permits unrestricted use, distribution, and reproduction in any medium, provided the original work is properly cited.

\begin{abstract}
When facing to make a portfolio decision, investors may care more about every portfolio's performance on a return and risk tradeoff. In this paper, a new low partial moment measurement that only punishes the loss risk is defined for selection variables based on L-S integral. Furthermore, a new performance measure for portfolio evaluation is proposed to generalize the Sharpe ratio in the fuzzy context. With the optimal performance criterion, a new parametric Sharpe ratio portfolio optimization model is developed wherein uncertain returns are presented as parametric interval-valued fuzzy variables. To make the proposed model easy to solve, we transform the fractional programming into an equivalent form and solve it with domain decomposition method (DDM). Finally, we apply the proposed performance measure into a portfolio selection problem, compare the computational results in different cases, and analyze the influence of different parameters on the optimal portfolio.
\end{abstract}

\section{Introduction}

Markowitz's seminal paper on portfolio selection [1] has a major impact on financial research field and introduced a theory popularly referred to as modern portfolio theory. The core of the theory is telling us the truth that investors should consider the risk and return together and determine the allocations of funds among investment alternatives on the basis of the trade-off between them [2]. In the past three decades, numerous researchers had contributed to the development of modern portfolio theory and proposed many optimization models and selection approaches for portfolio selection problem based on the trade-off analysis between reward and risk; see [3-7].

The Sharpe ratio $[8,9]$, also called reward-to-risk ratio, is often used to measure the risk-adjusted return of an investment asset or a portfolio. From the ratio, investors can distinguish how well the return of a portfolio compensates them for the risk taken. The expression of conventional Sharpe ratio was defined as the mean of excess return divided by its variance; therein the excess return is the differential return between a portfolio and the benchmark.
A critique to this measure is that variance treats the upside and downside equally as risks and penalizes volatility with respect to expected value in both directions. However, positive excess returns (i.e., windfall profits) should not be regarded as something to avoid by investors. The occurrence of the low partial moment (LPM) $[10,11]$ is the development of downside risk measure in the portfolio research, and it also represents the attitude of decision maker from seeking to risk neutral to risk aversion. After that, many scholars have modified the Sharpe ratio with the LPM and presented a series of risk-adjusted performance measures. Sortino and van der Meer [12] presented the Sortino ratio as a variation of Sharpe ratio that adjusted the expected return for the risk of falling short of the risk-free return. Keating and Shadwick [13] proposed the Omega function as a natural performance measure to analyze returns distributions with the risk of the downside, lower partial moment, and gain-loss. Kaplan and Knowles [14] developed a Kappa ratio as a generalized downside risk-adjusted performance measurement based on the concept of LPM with parameter $n$. The three rewardto-risk ratios are all based on the partial moments, and the difference of them is Omega; Sortino and Kappa ratio use 
LPMs of orders 1, 2, and n, respectively. Value-at-Risk (VaR) and conditional Value-at-Risk (CVaR) are two downside risk measurements which directly gauge the investors' losses. As an alternative risk measure, VaR [15] was incorporated into the ratio of the performance evaluation as the $\mathrm{VaR}$ ratio. Martin et al. [16] presented the CVaR ratio in which CVaR was substituted for variance. Chow and Lai [17] introduced the conditional Sharpe ratios (CSR) that measured lower partial risk-adjusted excess returns of an asset with respect to return distribution on the benchmark based on the conditional stochastic dominance. The authors summarized the monotonic transformations of Sharpe ratio with eleven downside risks in [18].

It is worth noting that, in real life, the future asset return sometimes needs to be given by the expert's judgement and estimation for no sufficient historical data or the noisy original data. Therefore, many scholars tried to model the uncertain parameter with fuzzy uncertainty [19-22], and a number of researchers have developed some downside risk measures in fuzzy decision problem $[23,24]$. Since the ability to measure the risk-adjusted performance of a portfolio, the Sharpe ratio was introduced to assess the portfolio in fuzzy circumstance. Hasuike and Katagiri [25] considered a random fuzzy portfolio selection problem with general distributions and transformed it into the deterministic standard mathematical programming problem by introducing Sharpe ratio and fuzzy goals. Raei and Jahromi [26] proposed a model based on multiple criteria decision making and then by implementation of fuzzy analytical network analysis found appropriate weights of the asset. To measure the efficiency of the proposed model, the Sharpe ratio was computed for performance evaluation of the optimal portfolio. Based on [27], Nguyen et al. [28] modeled stock returns by the LR triangular fuzzy random variables and introduced a portfolio performance ratio named "reward-to-uncertainty" ratio to measure a portfolio performance.

As the authors pointed in $[29,30]$, a common feature of most related studies is to assume the specific distributions of uncertain parameters known. However, in the financial market, the fully distributional information about the uncertain return affected by many factors is not always available. For this reason, it is reasonable to consider a more effective and flexible way to describe the uncertainty on return. Based on fuzzy possibility theory [31], Y. Liu and Y.-K. Liu [32] proposed the concept of parametric interval-valued fuzzy variable, which can handle the case of uncertainty contained in fuzzy possibility distribution. Specifically, the distribution of parametric interval-valued fuzzy variable is a variable possibility distribution. When the exact distribution of uncertain return is unavailable, the depictive approach of variable possibility distribution that depends on the choice of the parameter's value is valid.

Therefore, in the present paper, we consider a portfolio optimization problem in an incomplete market where there are multiple risk assets and one risk-free asset. We use a parametric optimization method to deal with portfolio performance evaluation; the uncertain return rate is characterized by variable possibility distribution and presented as a parametric interval-valued fuzzy variable. Based on L-S integral [33], we propose a new risk measure which meets the nature requirement only punishing the real risk which brings loss. We, therefore, modify the Sharpe ratio and formulate a parametric Sharpe ratio (PSR) to assess a portfolio in the fuzzy context. Subsequently, we proposed a fuzzy Sharpe ratio portfolio selection model with the viewpoint of portfolio performance evaluation. We also analyze the sensibility of parameters in the model and give a numerical example to illustrate the efficiency of the proposed model.

The rest of this paper is organized as follows. For a better understanding, in Section 2, we will briefly introduce the necessary knowledge about parametric interval-valued fuzzy variable and its selection variable. Then, based on L-S integral, the semimoment of selection variable is presented. According to the new risk measure, we refine the Sharpe ratio and a fuzzy parametric Sharpe ratio portfolio selection model will be developed in Section 3. In Section 4, we will derive the formulas of semimoment of excess return under the assumption that uncertain returns are parametric interval-valued trapezoidal/triangular fuzzy variables. After that, we analyze the proposed model, and the equivalent parametric forms of the model will be presented in Section 5. In Section 6, we will design a domain decomposition method to solve the model. For the sake of illustration, in Section 7, the application of the model will be discussed by a numerical example, and then we study the results with parameter analysis. Finally, some concluding remarks will be given in Section 8 .

\section{The Semimoment of Selection Variable}

Let $\left(\Gamma, \mathscr{P}(\Gamma), \widetilde{P}_{0 s}\right)$ be a fuzzy possibility space [31]. A map $\widetilde{\xi}=$ $\left(\widetilde{\xi}_{1}, \widetilde{\xi}_{2}, \ldots, \widetilde{\xi}_{n}\right): \Gamma \mapsto \Re^{n}$ is called an $n$-ary type 2 (T2) fuzzy vector. When $n=1$, the map $\widetilde{\xi}$ is called a T2 fuzzy variable.

The secondary possibility distribution function $\widetilde{\mu}_{\xi}(x)$ of $\xi$ is defined as

$$
\widetilde{\mu}_{\tilde{\xi}}(x)=\widetilde{P}_{0}\{\gamma \in \Gamma \mid \widetilde{\xi}(\gamma)=x\}, \quad x \in \mathfrak{R}^{n},
$$

and type 2 possibility distribution function $\mu_{\tilde{\xi}}(x, u)$ of $\xi$ is defined as

$$
\mu_{\tilde{\xi}}(x, u)=\operatorname{Pos}\left\{\tilde{\mu}_{\tilde{\xi}}(x)=u\right\}, \quad(x, u) \in \mathfrak{R}^{n} \times J_{x},
$$

where $J_{x} \subset[0,1]$ is the support of $\widetilde{\mu}_{\tilde{\xi}}(x)$.

As a special case of T2 fuzzy variable, if for any $x \in \mathfrak{R}, u \in$ $J_{x} \subseteq[0,1]$, the $\mathrm{T} 2$ possibility distribution function $\mu_{\tilde{\xi}}(x, u)=$ 1 , then $\widetilde{\xi}$ is an interval T2 fuzzy variable [32].

Furthermore, if the secondary possibility distribution $\widetilde{\mu}_{\tilde{\xi}}(x)$ is a single subinterval of $[0,1]$, which means if for any $x \in \Re, \widetilde{\mu}_{\tilde{\xi}}(x)$ is a subinterval $\left[\mu_{\xi^{L}}\left(x ; \theta_{l}\right), \mu_{\xi U}\left(x ; \theta_{r}\right)\right]$ of $[0,1]$ with parameters $\theta_{l}, \theta_{r} \in[0,1]$, then $\tilde{\xi}$ is called a parametric interval-valued fuzzy variable [32]

If $\theta_{l}=\theta_{r}=0$, then the reduced secondary possibility distribution is called the principle possibility distribution of $\widetilde{\xi}$, and the fuzzy variable associated with the principle possibility distribution is denoted by $\xi^{p}[34]$. 
Let $r_{1}<r_{2} \leq r_{3}<r_{4}$ be real numbers. Then a map $\tilde{\xi}$ is called a parametric interval-valued trapezoidal fuzzy variable if its secondary possibility distribution $\widetilde{\mu} \widetilde{\xi}(r)$ is the subinterval

$$
\begin{aligned}
& {\left[\frac{r-r_{1}}{r_{2}-r_{1}}-\theta_{l} \min \left\{\frac{r-r_{1}}{r_{2}-r_{1}}, \frac{r_{2}-r}{r_{2}-r_{1}}\right\}, \frac{r-r_{1}}{r_{2}-r_{1}}\right.} \\
& \left.+\theta_{r} \min \left\{\frac{r-r_{1}}{r_{2}-r_{1}}, \frac{r_{2}-r}{r_{2}-r_{1}}\right\}\right]
\end{aligned}
$$

of $[0,1]$ for $r \in\left[r_{1}, r_{2}\right]$, the interval $[1,1]$ for $r \in\left[r_{2}, r_{3}\right]$, and the subinterval

$$
\begin{aligned}
& {\left[\frac{r_{4}-r}{r_{4}-r_{3}}-\theta_{l} \min \left\{\frac{r_{4}-r}{r_{4}-r_{3}}, \frac{r-r_{3}}{r_{4}-r_{3}}\right\}, \frac{r_{4}-r}{r_{4}-r_{3}}\right.} \\
& \left.\quad+\theta_{r} \min \left\{\frac{r_{4}-r}{r_{4}-r_{3}}, \frac{r-r_{3}}{r_{4}-r_{3}}\right\}\right]
\end{aligned}
$$

of $[0,1]$ for $x \in\left[r_{3}, r_{4}\right]$, where $\theta_{l}, \theta_{r} \in[0,1]$ are two parameters characterizing the degree of uncertainty that $\widetilde{\xi}$ takes on the value $r$. We denote the parametric interval-valued trapezoidal fuzzy variable $\widetilde{\xi}$ with the above distribution by $\left[r_{1}, r_{2}, r_{3}, r_{4} ; \theta_{l}, \theta_{r}\right]$.

Let $r_{1}<r_{2}<r_{3}$ be real numbers. Then a map $\tilde{\xi}$ is called a parametric interval-valued triangular fuzzy variable if its secondary possibility distribution $\widetilde{\mu}_{\tilde{\xi}}(r)$ is the parametric subinterval

$$
\begin{aligned}
& {\left[\frac{r-r_{1}}{r_{2}-r_{1}}-\theta_{l} \min \left\{\frac{r-r_{1}}{r_{2}-r_{1}}, \frac{r_{2}-r}{r_{2}-r_{1}}\right\}, \frac{r-r_{1}}{r_{2}-r_{1}}\right.} \\
& \left.+\theta_{r} \min \left\{\frac{r-r_{1}}{r_{2}-r_{1}}, \frac{r_{2}-r}{r_{2}-r_{1}}\right\}\right]
\end{aligned}
$$

of $[0,1]$ for $r \in\left[r_{1}, r_{2}\right]$ and the parametric subinterval

$$
\begin{aligned}
& {\left[\frac{r_{3}-r}{r_{3}-r_{2}}-\theta_{l} \min \left\{\frac{r_{3}-r}{r_{3}-r_{2}}, \frac{r-r_{2}}{r_{3}-r_{2}}\right\}, \frac{r_{3}-r}{r_{3}-r_{2}}\right.} \\
& \left.+\theta_{r} \min \left\{\frac{r_{3}-r}{r_{3}-r_{2}}, \frac{r-r_{2}}{r_{3}-r_{2}}\right\}\right]
\end{aligned}
$$

of $[0,1]$ for $r \in\left[r_{2}, r_{3}\right]$, where $\theta=\left(\theta_{l}, \theta_{r}\right)$, and $\theta_{l}, \theta_{r} \in[0,1]$ are two parameters characterizing the degree of uncertainty that $\tilde{\xi}$ takes on the value $r$. For simplicity, we denote the parametric interval-valued triangular fuzzy variable $\tilde{\xi}$ with the above distribution by $\left[r_{1}, r_{2}, r_{3} ; \theta_{l}, \theta_{r}\right]$.

A fuzzy variable $\xi$ is called $\lambda$ selection variable of parametric interval-valued fuzzy variable $\tilde{\xi}$ [32], if $\xi$ has the following parametric possibility distribution function:

$$
\begin{aligned}
& \mu_{\xi}(r ; \theta)=(1-\lambda) \mu_{\xi^{L}}\left(r ; \theta_{l}\right)+\lambda \mu_{\xi^{U}}\left(r ; \theta_{r}\right), \\
& \lambda \in[0,1],
\end{aligned}
$$

where $\theta=\left(\theta_{l}, \theta_{r}\right)$.

If $\xi$ is a selection variable with finite expected value $m$, the definition of $(\xi-m)^{-}$is

$$
(\xi-m)^{-}= \begin{cases}m-\xi, & \text { if } \xi \leq m \\ 0, & \text { if } \xi>m .\end{cases}
$$

Based on L-S integral [33], we define the semimoment of $\xi$ as follows.

Definition 1. If $\xi$ is a selection variable with finite expected value $m$, then the integral

$$
\begin{aligned}
\operatorname{SM}[\xi] & =\int_{(-\infty,+\infty)}\left[(\xi-m)^{-}\right]^{2} \mathrm{~d}(\operatorname{Cr}\{\xi \leq r\}) \\
& =\int_{(-\infty, m)}(m-\xi)^{2} \mathrm{~d}(\operatorname{Cr}\{\xi \leq r\}),
\end{aligned}
$$

is called the semimoment of $\xi$, where $\operatorname{Cr}\{\xi \leq r\}$ is the credibility distribution of $\xi$.

The credibility distribution of $\xi$ is defined in [35] and computed by

$$
\operatorname{Cr}\{\xi \leq r\}=\frac{1}{2}\left(1+\sup _{t \leq r} \mu_{\xi}(t ; \theta)-\sup _{t>r} \mu_{\xi}(t ; \theta)\right) .
$$

\section{A Parametric Sharpe Ratio Portfolio Optimization Model}

Assume an investor wants to make an allocation decision in a financial market, where there are $n$ risk assets/securities and one risk-free asset. The investor wants a portfolio of the $n$ risk assets plus the risk-free asset based on the portfolio performance ratio.

The return of the risk-free asset is denoted by $r_{f}$, and the investor can deposit his/her money or invest in treasury bond at this rate. Usually, $r_{f}$ is as a benchmark to the returns of risky assets. That means the investor demands a premium over the risk-free return by investing in risky assets, and the risk-free return is often less than risk return. If a risk-free asset performs better than the risky assets, it is advisable for investor to put all money on it.

For various economic and noneconomic factors, the returns of risky assets are uncertain and their fixed possibility distributions are difficultly obtained in many situations. Thus, in this paper, we consider the case that the uncertain return of the $i$ th risky asset is presented as a parametric interval-valued fuzzy variable $\tilde{\xi}_{i}(i=1,2, \ldots, n)$, and the uncertainty return vector is $\widetilde{\xi}=\left(\widetilde{\xi}_{1}, \ldots, \widetilde{\xi}_{n}\right)^{T}$.

Let $x_{i}$ be the percentage of wealth invested in the $i$ th risky asset, and $\mathbf{x}=\left(x_{1}, \ldots, x_{n}\right)^{T}$ is the weight vector of a portfolio. Note that $\mathbf{x}$ does not have to sum to 1 , and the remaining part $1-\mathbf{x}^{T} \mathbf{1}$ can be invested in the risk-free asset, where $\mathbf{1}$ is an $n$ dimension column vector and every component is 1 . If shortselling does not exist in this asset market, the investment proportion $\mathbf{x} \geq 0$. This is a common restriction on portfolio weights. In practice, for investment policy or legal reasons, or sometimes just because of particular asset classes, the investor cannot sell assets short.

For a portfolio $\mathbf{x}$, the total return $r_{p}$ is equal to the sum of the returns of two kinds of assets; that is,

$$
r_{p}=\mathbf{x}^{T} \widetilde{\xi}+\left(1-\mathbf{x}^{T} \mathbf{1}\right) r_{f} .
$$


Using the risk-free return $r_{f}$ as the benchmark, the excess return is

$$
r_{p}-r_{f}=\mathbf{x}^{T} \widetilde{\xi}+\left(1-\mathbf{x}^{T} \mathbf{1}\right) r_{f}-r_{f}=\mathbf{x}^{T} \widetilde{\xi}-r_{f} \mathbf{x}^{T} \mathbf{1} .
$$

Because $\tilde{\xi}$ is a parametric interval-valued fuzzy return vector, the excess return rate of a portfolio is a linear combination of parametric interval-valued fuzzy variables. According to [32], $\mathbf{x}^{T} \widetilde{\xi}-r_{f} \mathbf{x}^{T} \mathbf{1}$ is still a parametric intervalvalued fuzzy variable. The $\lambda$ selection of the excess return rate is denoted as $\mathbf{x}^{T} \xi-r_{f} \mathbf{x}^{T} \mathbf{1}$. Then the portfolio's excess expected return rate is $E\left[\mathbf{x}^{T} \xi-r_{f} \mathbf{x}^{T} \mathbf{1}\right]$.

To make a best trade-off between risk and return, the core problem is to find an appropriate risk measure according to the risk attitudes of different investors. In this paper, we use semimoment to measure the risk, and the semimoment of a portfolio's excess return rate is $\operatorname{SM}\left[\mathbf{x}^{T} \xi-r_{f} \mathbf{x}^{T} \mathbf{1}\right]$.

In general, the population Sharpe ratio (SR) formula is demonstrated by

$$
\mathrm{SR}=\frac{\text { excess expected return }}{\text { unit risk deviation }} .
$$

With the expression above, we can formally define a new performance measure, that is, the fuzzy parametric Sharpe ratio (PSR) of excess return rate $\mathbf{x}^{T} \xi-r_{f} \mathbf{x}^{T} \mathbf{1}$, as follows:

$$
\operatorname{PSR}(\mathbf{x}, \xi ; \lambda)=\frac{E\left[\mathbf{x}^{T} \xi-r_{f} \mathbf{x}^{T} \mathbf{1}\right]}{\operatorname{SM}\left[\mathbf{x}^{T} \xi-r_{f} \mathbf{x}^{T} \mathbf{1}\right]} .
$$

According to the different risk attitudes, there exist two optimization directions. We can choose maximizing the excess expected return per unit of risk or minimizing the risk per unit of return. In the present paper, we take the viewpoint of the investor who is willing to minimize the risk per unit of return. Thus, minimizing fuzzy parametric Sharpe ratio of excess return rate $\mathbf{x}^{T} \xi-r_{f} \mathbf{x}^{T} \mathbf{1}$ is expressed as

$$
\min _{\mathbf{x}} \operatorname{PSR}(\mathbf{x}, \xi ; \lambda)=\frac{\operatorname{SM}\left[\mathbf{x}^{T} \xi-r_{f} \mathbf{x}^{T} \mathbf{1}\right]}{E\left[\mathbf{x}^{T} \xi-r_{f} \mathbf{x}^{T} \mathbf{1}\right]} .
$$

For a portfolio $\mathbf{x}, \mathbf{x}^{T} \widetilde{\xi}$ is a parametric interval-valued fuzzy variable and $r_{f} \mathbf{x}^{T} \mathbf{1}$ is a constant. If $\mathbf{x}^{T} \xi$ is the $\lambda$ selection of $\mathbf{x}^{T} \widetilde{\xi}$, by the definitions of $\lambda$ selection and semimoment, we have

$$
\begin{aligned}
E\left[\mathbf{x}^{T} \xi-r_{f} \mathbf{x}^{T} \mathbf{1}\right] & =E\left[\mathbf{x}^{T} \xi\right]-r_{f} \mathbf{x}^{T} \mathbf{1}, \\
\operatorname{SM}\left[\mathbf{x}^{T} \xi-r_{f} \mathbf{x}^{T} \mathbf{1}\right] & =\operatorname{SM}\left[\mathbf{x}^{T} \xi\right] .
\end{aligned}
$$

Thus, under the assumption of no short-selling, a fuzzy parametric Sharpe ratio portfolio selection model can be built as follows:

$$
\begin{aligned}
\min & \frac{\mathrm{SM}\left[\mathbf{x}^{T} \xi\right]}{E\left[\mathbf{x}^{T} \xi\right]-r_{f} \mathbf{x}^{T} \mathbf{1}} \\
\text { subject to } & \mathbf{x}^{T} \mathbf{1} \leq 1 \\
& x_{i} \geq 0, \quad i=1,2, \ldots, n .
\end{aligned}
$$

Model (17) is a fractional programming; the complexity of solution mainly focuses on the computation of the objective function. In the following section, we will discuss how to deal with this computational issue.

\section{The Analytic Expression of Semimoment}

In this section, we focus on the computational issue of Sharpe ratio of excess return rate. Under the assumption that the uncertain returns are some special parametric intervalvalued fuzzy variables, we present the determinate equivalent parametric forms of semimoment and expected value.

Suppose a fuzzy return vector is $\widetilde{\xi}=\left(\widetilde{\xi}_{1}, \ldots, \widetilde{\xi}_{n}\right)^{T}$, where $\tilde{\xi}_{i}=\left[r_{1 i}, r_{2 i}, r_{3 i}, r_{4 i} ; \theta_{l i}, \theta_{r i}\right]$ are parametric interval-valued trapezoidal fuzzy variables and $\mathbf{x}=\left(x_{1}, \ldots, x_{n}\right)^{T}$ is the portfolio's weight vector, $x_{i} \in[0,1]$. Then the coefficient matrix of the fuzzy return vector is presented as

$$
C=\left(\begin{array}{cccc}
r_{11} & r_{21} & r_{31} & r_{41} \\
r_{12} & r_{22} & r_{32} & r_{42} \\
\cdots & \cdots & \cdots & \cdots \\
r_{1 n} & r_{2 n} & r_{3 n} & r_{4 n}
\end{array}\right)_{n \times 4}
$$

and denote $c_{j}=\left(r_{j 1}, \ldots, r_{j n}\right)^{T}, j=1,2,3,4$. Obviously, we have $C=\left(c_{1}, c_{2}, c_{3}, c_{4}\right)$.

By [32], the expected value of fuzzy return $x^{T} \xi$ is

$$
\begin{aligned}
E\left[\mathbf{x}^{T} \xi\right] & \\
= & \frac{1}{4} \sum_{i=1}^{n} x_{i}\left(r_{1 i}+r_{2 i}+r_{3 i}+r_{4 i}\right) \\
& +\frac{1}{8}\left[\lambda \theta_{r}-(1-\lambda) \theta_{l}\right] \sum_{i=1}^{n} x_{i}\left(r_{1 i}-r_{2 i}-r_{3 i}+r_{4 i}\right) \\
= & \frac{1}{4} \mathbf{x}^{T}\left(c_{1}+c_{2}+c_{3}+c_{4}\right) \\
& +\frac{1}{8}\left[\lambda \theta_{r}-(1-\lambda) \theta_{l}\right] \mathbf{x}^{T}\left(c_{1}-c_{2}-c_{3}+c_{4}\right),
\end{aligned}
$$

where parameters $\theta_{l}=\max _{1 \leq j \leq n} \theta_{l j}$ and $\theta_{r}=\min _{1 \leq j \leq n} \theta_{r j}$.

Denoting $E\left[\mathbf{x}^{T} \xi\right]=m$, we can draw the following conclusions of semimoment.

Theorem 2. Let the uncertain return of asset $i$ be parametric interval-valued trapezoidal fuzzy variable $\widetilde{\xi}_{i}=\left[r_{1 i}, r_{2 i}, r_{3 i}\right.$, $\left.r_{4 i} ; \theta_{l i}, \theta_{r i}\right]$ and $x_{i}$ real numbers for $i=1,2, \ldots, n$. If the principle possibility distributions of $\widetilde{\xi}_{i}$ are mutually independent, then the semimoment of fuzzy return sum $\mathbf{x}^{T} \xi$ is as follows.

(i) If $m<\mathbf{x}^{T} c_{2}$, then 


$$
S M\left[\mathbf{x}^{T} \xi\right]=\frac{1}{2} r^{T} Q r-\frac{\left[1-\lambda \theta_{r}+(1-\lambda) \theta_{l}\right]\left\{2 \mathbf{x}^{T}\left(c_{1}+c_{2}-c_{3}-c_{4}\right)-\left[\lambda \theta_{r}-(1-\lambda) \theta_{l}\right] \mathbf{x}^{T}\left(c_{1}-c_{2}-c_{3}+c_{4}\right)\right\}^{3}}{3072 \mathbf{x}^{T}\left(c_{2}-c_{1}\right)}
$$

where $r=C^{T} \mathbf{x}=\left(\sum_{i=1}^{n} x_{i} r_{1 i}, \sum_{i=1}^{n} x_{i} r_{2 i}, \sum_{i=1}^{n} x_{i} r_{3 i}\right.$, $\left.\sum_{i=1}^{n} x_{i} r_{4 i}\right)^{T}$, and the elements of the symmetric matrix $Q$ include

$$
\begin{aligned}
Q_{11}= & \frac{1}{128}\left[\lambda \theta_{r}-(1-\lambda) \theta_{l}\right]^{3} \\
& -\frac{7}{128}\left[\lambda \theta_{r}-(1-\lambda) \theta_{l}\right]^{2} \\
& +\frac{7}{96}\left[\lambda \theta_{r}-(1-\lambda) \theta_{l}\right]+\frac{13}{96}
\end{aligned}
$$$$
Q_{12}=-\frac{1}{32}\left[\lambda \theta_{r}-(1-\lambda) \theta_{l}\right]^{3}
$$$$
+\frac{3}{32}\left[\lambda \theta_{r}-(1-\lambda) \theta_{l}\right]^{2}
$$$$
+\frac{1}{12}\left[\lambda \theta_{r}-(1-\lambda) \theta_{l}\right]+\frac{1}{24}
$$$$
Q_{13}=-\frac{1}{32}\left[\lambda \theta_{r}-(1-\lambda) \theta_{l}\right]^{3}
$$$$
+\frac{5}{32}\left[\lambda \theta_{r}-(1-\lambda) \theta_{l}\right]^{2}
$$$$
-\frac{1}{16}\left[\lambda \theta_{r}-(1-\lambda) \theta_{l}\right]+\frac{1}{4},
$$$$
Q_{14}=\frac{1}{32}\left[\lambda \theta_{r}-(1-\lambda) \theta_{l}\right]^{3}-\frac{1}{32}\left[\lambda \theta_{r}-(1-\lambda) \theta_{l}\right]^{2}
$$$$
-\frac{5}{16}\left[\lambda \theta_{r}-(1-\lambda) \theta_{l}\right]-\frac{1}{4},
$$$$
Q_{22}=\frac{1}{128}\left[\lambda \theta_{r}-(1-\lambda) \theta_{l}\right]^{3}
$$$$
+\frac{1}{128}\left[\lambda \theta_{r}-(1-\lambda) \theta_{l}\right]^{2}
$$$$
+\frac{1}{96}\left[\lambda \theta_{r}-(1-\lambda) \theta_{l}\right]+\frac{1}{96},
$$$$
Q_{23}=\frac{1}{32}\left[\lambda \theta_{r}-(1-\lambda) \theta_{l}\right]^{3}+\frac{7}{32}\left[\lambda \theta_{r}-(1-\lambda) \theta_{l}\right]^{2}
$$$$
+\frac{3}{16}\left[\lambda \theta_{r}-(1-\lambda) \theta_{l}\right]
$$$$
Q_{24}=-\frac{1}{32}\left[\lambda \theta_{r}-(1-\lambda) \theta_{l}\right]^{3}
$$$$
-\frac{3}{32}\left[\lambda \theta_{r}-(1-\lambda) \theta_{l}\right]^{2}
$$$$
-\frac{1}{16}\left[\lambda \theta_{r}-(1-\lambda) \theta_{l}\right]
$$

$$
\begin{aligned}
Q_{33}= & \frac{1}{128}\left[\lambda \theta_{r}-(1-\lambda) \theta_{l}\right]^{3} \\
& -\frac{3}{128}\left[\lambda \theta_{r}-(1-\lambda) \theta_{l}\right]^{2}+\frac{1}{32}, \\
Q_{34}= & -\frac{1}{32}\left[\lambda \theta_{r}-(1-\lambda) \theta_{l}\right]^{3} \\
& -\frac{1}{32}\left[\lambda \theta_{r}-(1-\lambda) \theta_{l}\right]^{2} \\
& +\frac{1}{8}\left[\lambda \theta_{r}-(1-\lambda) \theta_{l}\right]+\frac{1}{8}, \\
Q_{44}= & \frac{1}{128}\left[\lambda \theta_{r}-(1-\lambda) \theta_{l}\right]^{3} \\
& +\frac{5}{128}\left[\lambda \theta_{r}-(1-\lambda) \theta_{l}\right]^{2} \\
& +\frac{1}{16}\left[\lambda \theta_{r}-(1-\lambda) \theta_{l}\right]+\frac{1}{32} .
\end{aligned}
$$

(ii) If $\mathbf{x}^{T} \mathcal{c}_{2} \leq m<\mathbf{x}^{T} c_{3}$, then

$$
S M\left[\mathbf{x}^{T} \xi\right]=\frac{1}{2} r^{T} \operatorname{Pr}
$$

where $r=C^{T} \mathbf{x}=\left(\sum_{i=1}^{n} x_{i} r_{1 i}, \sum_{i=1}^{n} x_{i} r_{2 i}, \sum_{i=1}^{n} x_{i} r_{3 i}\right.$, $\left.\sum_{i=1}^{n} x_{i} r_{4 i}\right)^{T}$, and the elements of the symmetric matrix $P$ include

$$
\begin{aligned}
P_{11}= & -\frac{3}{64}\left[\lambda \theta_{r}-(1-\lambda) \theta_{l}\right]^{2}+\frac{1}{16}\left[\lambda \theta_{r}-(1-\lambda) \theta_{l}\right] \\
& +\frac{7}{48}, \\
P_{12}= & \frac{3}{16}\left[\lambda \theta_{r}-(1-\lambda) \theta_{l}\right]^{2}-\frac{1}{12}, \\
P_{13}= & P_{24}=\frac{1}{16}\left[\lambda \theta_{r}-(1-\lambda) \theta_{l}\right]^{2}-\frac{1}{4}, \\
P_{14}= & -\frac{1}{16}\left[\lambda \theta_{r}-(1-\lambda) \theta_{l}\right]^{2}-\frac{1}{4}\left[\lambda \theta_{r}-(1-\lambda) \theta_{l}\right] \\
& -\frac{1}{4}, \\
P_{22}= & -\frac{3}{64}\left[\lambda \theta_{r}-(1-\lambda) \theta_{l}\right]^{2}-\frac{1}{16}\left[\lambda \theta_{r}-(1-\lambda) \theta_{l}\right] \\
& -\frac{7}{48},
\end{aligned}
$$




$$
\begin{aligned}
P_{23}= & -\frac{1}{16}\left[\lambda \theta_{r}-(1-\lambda) \theta_{l}\right]^{2}+\frac{3}{4}\left[\lambda \theta_{r}-(1-\lambda) \theta_{l}\right] \\
& -\frac{1}{4}, \\
P_{33}= & \frac{1}{64}\left[\lambda \theta_{r}-(1-\lambda) \theta_{l}\right]^{2}-\frac{1}{16}\left[\lambda \theta_{r}-(1-\lambda) \theta_{l}\right] \\
& +\frac{1}{32},
\end{aligned}
$$$$
P_{34}=-\frac{1}{16}\left[\lambda \theta_{r}-(1-\lambda) \theta_{l}\right]^{2}+\frac{1}{4},
$$

$$
\begin{aligned}
P_{44}= & \frac{1}{64}\left[\lambda \theta_{r}-(1-\lambda) \theta_{l}\right]^{2}+\frac{1}{16}\left[\lambda \theta_{r}-(1-\lambda) \theta_{l}\right] \\
& +\frac{1}{32} .
\end{aligned}
$$

(iii) If $m \geq \mathbf{x}^{T} c_{3}$, then

$$
S M\left[\mathbf{x}^{T} \xi\right]=\frac{1}{2} r^{T} \operatorname{Pr}-\frac{\left[1-\lambda \theta_{r}+(1-\lambda) \theta_{l}\right]\left\{2 \mathbf{x}^{T}\left(c_{1}+c_{2}-3 c_{3}+c_{4}\right)-\left[\lambda \theta_{r}-(1-\lambda) \theta_{l}\right] \mathbf{x}^{T}\left(c_{1}-c_{2}-c_{3}+c_{4}\right)\right\}^{3}}{3072 \mathbf{x}^{T}\left(c_{4}-c_{3}\right)}
$$

where $r=C^{T} \mathbf{x}=\left(\sum_{i=1}^{n} x_{i} r_{1 i}, \sum_{i=1}^{n} x_{i} r_{2 i}, \sum_{i=1}^{n} x_{i} r_{3 i}\right.$, $\left.\sum_{i=1}^{n} x_{i} r_{4 i}\right)^{T}$, and the elements of the symmetric matrix $P$ are the same as case (ii), with the parameters $\theta_{l}=$ $\max _{1 \leq i \leq n} \theta_{l i}$ and $\theta_{r}=\min _{1 \leq i \leq n} \theta_{r i}$.

Moreover, the semimoment $S M\left[\mathbf{x}^{T} \xi\right]$ in case (ii) is a parametric quadratic convex function with respect to decision vector $x \in R^{n}$.

On the other side, if fuzzy returns $\widetilde{\xi}_{i}=\left[r_{1 i}, r_{2 i}, r_{3 i} ; \theta_{l i}, \theta_{r i}\right]$ are parametric interval-valued triangular fuzzy variables and $\mathbf{x}=\left(x_{1}, \ldots, x_{n}\right)^{T}$ is the portfolio's weight vector, $x_{i} \in[0,1]$, then the coefficient matrix of the fuzzy return vector is presented as

$$
C=\left(\begin{array}{ccc}
r_{11} & r_{21} & r_{31} \\
r_{12} & r_{22} & r_{32} \\
\cdots & \cdots & \cdots \\
r_{1 n} & r_{2 n} & r_{3 n}
\end{array}\right)_{n \times 3},
$$

and denote $c_{j}=\left(r_{j 1}, \ldots, r_{j n}\right)^{T}, j=1,2,3$. Obviously, we have $C=\left(c_{1}, c_{2}, c_{3}\right)$.
By [32], the expected value of $\lambda$ selection variable $\mathbf{x}^{T} \xi$ is

$$
\begin{aligned}
E\left[\mathbf{x}^{T} \xi\right] & \\
= & \frac{\sum_{i=1}^{n} x_{i}\left(r_{1 i}+2 r_{2 i}+r_{3 i}\right)}{4} \\
& +\frac{\left[\lambda \theta_{r}-(1-\lambda) \theta_{l}\right] \sum_{i=1}^{n} x_{i}\left(r_{1 i}-2 r_{2 i}+r_{3 i}\right)}{8} \\
= & \frac{\mathbf{x}^{T}\left(c_{1}+2 c_{2}+c_{3}\right)}{4} \\
& +\frac{\left[\lambda \theta_{r}-(1-\lambda) \theta_{l}\right] \mathbf{x}^{T}\left(c_{1}-2 c_{2}+c_{3}\right)}{8},
\end{aligned}
$$

where the parameters $\theta_{l}=\max _{1 \leq j \leq n} \theta_{l j}$ and $\theta_{r}=\min _{1 \leq j \leq n} \theta_{r j}$. Denoting $E\left[\mathbf{x}^{T} \xi\right]=m$, we have the following corollary.

Corollary 3. Let the uncertain return of asset $i$ be parametric interval-valued triangular fuzzy variable $\widetilde{\xi}_{i}=\left[r_{1 i}, r_{2 i}, r_{3 i}\right.$; $\left.\theta_{l i}, \theta_{r i}\right]$ and $x_{i}$ real numbers for $i=1,2, \ldots, n$. If the principle possibility distributions of $\widetilde{\xi}_{i}$ are mutually independent, then the semimoment of fuzzy return $\operatorname{sum} \mathbf{x}^{T} \xi$ is as follows.

(i) If $m<\mathbf{x}^{T} c_{2}$, then

$$
S M\left[\mathbf{x}^{T} \xi\right]=\frac{1}{2} r^{T} R r-\frac{\left[1-\lambda \theta_{r}+(1-\lambda) \theta_{l}\right]\left\{2 \mathbf{x}^{T}\left(c_{1}-c_{3}\right)-\left[\lambda \theta_{r}+(1-\lambda) \theta_{l}\right] \mathbf{x}^{T}\left(c_{1}-2 c_{2}+c_{3}\right)\right\}^{3}}{3072 \mathbf{x}^{T}\left(c_{2}-c_{1}\right)}
$$

where $r=C^{T} \mathbf{x}=\left(\sum_{i=1}^{n} x_{i} r_{1 i}, \sum_{i=1}^{n} x_{i} r_{2 i}, \sum_{i=1}^{n} x_{i} r_{3 i}\right)^{T}$, and the elements of the symmetric matrix $R$ include

$$
\begin{aligned}
R_{11}= & \frac{1}{128}\left[\lambda \theta_{r}-(1-\lambda) \theta_{l}\right]^{3} \\
& -\frac{7}{128}\left[\lambda \theta_{r}-(1-\lambda) \theta_{l}\right]^{2}
\end{aligned}
$$

$$
\begin{aligned}
& +\frac{7}{96}\left[\lambda \theta_{r}-(1-\lambda) \theta_{l}\right]+\frac{13}{96} \\
R_{12}= & -\frac{1}{16}\left[\lambda \theta_{r}-(1-\lambda) \theta_{l}\right]^{3}+\frac{1}{4}\left[\lambda \theta_{r}-(1-\lambda) \theta_{l}\right]^{2} \\
& +\frac{1}{48}\left[\lambda \theta_{r}-(1-\lambda) \theta_{l}\right]-\frac{7}{24}
\end{aligned}
$$




$$
\begin{aligned}
R_{13}= & \frac{1}{32}\left[\lambda \theta_{r}-(1-\lambda) \theta_{l}\right]^{3}-\frac{1}{32}\left[\lambda \theta_{r}-(1-\lambda) \theta_{l}\right]^{2} \\
& -\frac{5}{16}\left[\lambda \theta_{r}-(1-\lambda) \theta_{l}\right]-\frac{1}{4}, \\
R_{22}= & \frac{1}{32}\left[\lambda \theta_{r}-(1-\lambda) \theta_{l}\right]^{3}-\frac{1}{32}\left[\lambda \theta_{r}-(1-\lambda) \theta_{l}\right]^{2} \\
& -\frac{1}{48}\left[\lambda \theta_{r}-(1-\lambda) \theta_{l}\right]+\frac{1}{24}, \\
R_{23}= & -\frac{1}{16}\left[\lambda \theta_{r}-(1-\lambda) \theta_{l}\right]^{3}-\frac{1}{8}\left[\lambda \theta_{r}-(1-\lambda) \theta_{l}\right]^{2}
\end{aligned}
$$

$$
\begin{aligned}
& +\frac{1}{16}\left[\lambda \theta_{r}-(1-\lambda) \theta_{l}\right]+\frac{1}{8} \\
R_{33}= & \frac{1}{128}\left[\lambda \theta_{r}-(1-\lambda) \theta_{l}\right]^{3} \\
& +\frac{5}{128}\left[\lambda \theta_{r}-(1-\lambda) \theta_{l}\right]^{2} \\
& +\frac{1}{16}\left[\lambda \theta_{r}-(1-\lambda) \theta_{l}\right]+\frac{1}{32}
\end{aligned}
$$

(ii) If $m \geq \mathbf{x}^{T} c_{2}$, then

$$
S M\left[\mathbf{x}^{T} \xi\right]=\frac{1}{2} r^{T} S r-\frac{\left[1-\lambda \theta_{r}+(1-\lambda) \theta_{l}\right]\left[2+\lambda \theta_{r}-(1-\lambda) \theta_{l}\right]^{3}\left[\mathbf{x}^{T}\left(c_{1}-2 c_{2}+c_{3}\right)\right]^{3}}{3072 \mathbf{x}^{T}\left(c_{3}-c_{2}\right)}
$$

where $r=C^{T} \mathbf{x}=\left(\sum_{i=1}^{n} x_{i} r_{1 i}, \sum_{i=1}^{n} x_{i} r_{2 i}, \sum_{i=1}^{n} x_{i} r_{3 i}\right)^{T}$, and the elements of the symmetric matrix $S$ include

$$
\begin{aligned}
S_{11}= & -\frac{3}{64}\left[\lambda \theta_{r}-(1-\lambda) \theta_{l}\right]^{2}+\frac{1}{16}\left[\lambda \theta_{r}-(1-\lambda) \theta_{l}\right] \\
& +\frac{7}{48}, \\
S_{12}= & \frac{1}{4}\left[\lambda \theta_{r}-(1-\lambda) \theta_{l}\right]^{2}-\frac{1}{3}, \\
S_{13}= & -\frac{1}{16}\left[\lambda \theta_{r}-(1-\lambda) \theta_{l}\right]^{2}-\frac{1}{4}\left[\lambda \theta_{r}-(1-\lambda) \theta_{l}\right] \\
& -\frac{1}{4}, \\
S_{22}= & 2-\frac{1}{16}\left[\lambda \theta_{r}-(1-\lambda) \theta_{l}\right]^{2}+\frac{1}{12}, \\
S_{23}= & 0, \\
S_{33}= & \frac{1}{64}\left[\lambda \theta_{r}-(1-\lambda) \theta_{l}\right]^{2}+\frac{1}{16}\left[\lambda \theta_{r}-(1-\lambda) \theta_{l}\right] \\
& +\frac{1}{16},
\end{aligned}
$$

with the parameters $\theta_{l}=\max _{1 \leq i \leq n} \theta_{l i}$ and $\theta_{r}=\min _{1 \leq i \leq n} \theta_{r i}$.

\section{The Equivalent Parametric Programming}

In this section, we firstly illustrate how the fractional formulation of fuzzy parametric Sharpe ratio portfolio selection model can be transformed into an equivalent nonlinear form and then derive its equivalent parametric submodels.

5.1. The Transformation of Fuzzy Sharpe Ratio Model. In mathematical programming, if fractional programming meets the four conditions
(A1) the feasible region $\mathscr{B}$ is bounded and nonempty;

(A2) for every $x \in \mathscr{B}, g(x)>0$;

(A3) for every $x \in \mathscr{B}, f(x)>0$;

(A4) $g(x)$ is a continuous and positively homogeneous risk measure, then fractional programming

$$
\min \frac{g(x)}{f(x)}
$$

subject to $x \in \mathscr{B}$

can be transformed into easily solving programming

$$
\begin{array}{cl}
\min & g(y, t) \\
\text { subject to } & f(y, t) \geq 1 \\
& A y-b t=0 \\
& y \geq 0 \\
& t>0,
\end{array}
$$

where $t=1 / f(x)>0$ and $y=t x$ (the Charnes-Cooper transformation [36]).

Subsequently, we will check the conditions of model (17).

(A1) $\mathbf{x}$ is the weight vector of a portfolio, and $x_{i} \geq 0, i=$ $1,2, \ldots, n$, so $\mathscr{B}$ is bounded and not negative.

(A2) For any $\mathbf{x}$, obviously, $g(\mathbf{x})=\operatorname{SM}\left[\mathbf{x}^{T} \xi\right]>0$.

(A3) The aim of investment is to gain the high return, so the return of risk asset should be higher than that of risk-free asset; namely, for any $\mathbf{x}, E\left[\mathbf{x}^{T} \xi\right]-r_{f} \mathbf{x}^{T} \mathbf{1}>0$.

(A4) By the definition of semimoment, $g(\mathbf{x})=\operatorname{SM}\left[\mathbf{x}^{T} \xi\right]$ is continuous and positive homogeneous. 
So far, we verify that the model meets four terms, and that means model (17) can be translated to its equivalent form:

$$
\begin{array}{ll}
\min & \operatorname{SM}\left[\mathbf{y}^{T} \xi\right] \\
\text { subject to } & E\left[\mathbf{y}^{T} \xi\right]-r_{f} \mathbf{y}^{T} \mathbf{1}-1 \geq 0 \\
& \mathbf{y}^{T} \mathbf{1}-t \leq 0 \\
& y_{i} \geq 0, \quad i=1,2, \ldots, n \\
& t>0 .
\end{array}
$$

According to [37], we can derive the relationship of optimal solutions between model (17) and model (33) easily. The decision variable of model (17) is $\mathbf{x} \in R^{n}$, while the decision variables of model (33) are $\mathbf{y} \in R^{n}$ and $t$. If $\left(\mathbf{y}^{*}, t^{*}\right) \in$ $R^{n+1}$ is a globally optimal solution for model (33), then $\mathbf{x}^{*}=$ $\mathbf{y}^{*} / t^{*}$ is a globally optimal solution for model (17).
5.2. The Equivalent Parametric Submodels. In the previous subsection, fuzzy Sharpe ratio portfolio selection model (17) has been transformed into equivalent nonfractional programming (33). In the following, we will discuss the equivalent parametric submodels of model (33) under the assumption that uncertain returns are parametric intervalvalued trapezoidal fuzzy variables.

Based on Theorem 2 and (19), if $E\left[\mathbf{x}^{T} \xi\right]<\mathbf{x}^{T} c_{2}$, we have

$$
E\left[\mathbf{x}^{T} \xi\right]-r_{f} \mathbf{x}^{T} \mathbf{1}<\mathbf{x}^{T} c_{2}-r_{f} \mathbf{x}^{T} \mathbf{1}
$$

Let $E\left[\mathbf{x}^{T} \xi\right]-r_{f} \mathbf{x}^{T} \mathbf{1}=1 / t$, by the discussion in Section 5.1; then $\mathbf{y}^{T} c_{2}-r_{f} \mathbf{y}^{T} \mathbf{1}>1$.

Thus, if $E\left[\mathbf{x}^{T} \xi\right]<\mathbf{x}^{T} c_{2}$, that is, $\mathbf{y}^{T} c_{2}-r_{f} \mathbf{y}^{T} \mathbf{1}>1$, we can obtain the equivalent parametric programming of model (33) as follows:

$$
\begin{aligned}
\min & \frac{1}{2} \mathbf{y}^{T} C C^{T} \mathbf{y}-\frac{\left[1-\lambda \theta_{r}+(1-\lambda) \theta_{l}\right]\left\{2 \mathbf{y}^{T}\left(c_{1}+c_{2}-c_{3}-c_{4}\right)-\left[\lambda \theta_{r}-(1-\lambda) \theta_{l}\right] \mathbf{y}^{T}\left(c_{1}-c_{2}-c_{3}+c_{4}\right)\right\}^{3}}{3072 \mathbf{y}^{T}\left(c_{2}-c_{1}\right)} \\
\text { subject to } & \frac{\mathbf{y}^{T}\left(c_{1}+c_{2}+c_{3}+c_{4}\right)}{4}+\frac{\left[\lambda \theta_{r}-(1-\lambda) \theta_{l}\right] \mathbf{y}^{T}\left(c_{1}-c_{2}-c_{3}+c_{4}\right)}{8}-r_{f} \mathbf{y}^{T} \mathbf{1}-1 \geq 0 \\
& \mathbf{y}^{T} c_{2}>1+r_{f} \mathbf{y}^{T} \mathbf{1} \\
& \mathbf{y}^{T} \mathbf{1}-t \leq 0 \\
& y_{i} \geq 0, \quad i=1,2, \ldots, n \\
& t>0
\end{aligned}
$$

Analogously, if $\mathbf{x}^{T} c_{2} \leq E\left[\mathbf{x}^{T} \xi\right]<\mathbf{x}^{T} c_{3}$, that is, $\mathbf{y}^{T} c_{2} \leq 1+$ $r_{f} \mathbf{y}^{T} \mathbf{1}<\mathbf{y}^{T} c_{3}$, then model (33) can be transformed into the following equivalent parametric programming:

$$
\begin{aligned}
\min \frac{1}{2} \mathbf{y}^{T} C P C^{T} \mathbf{y} \\
\text { subject to } \frac{\mathbf{y}^{T}\left(c_{1}+c_{2}+c_{3}+c_{4}\right)}{4} \\
\\
+\frac{\left[\lambda \theta_{r}-(1-\lambda) \theta_{l}\right] \mathbf{y}^{T}\left(c_{1}-c_{2}-c_{3}+c_{4}\right)}{8} \\
-r_{f} \mathbf{y}^{T} \mathbf{1}-1 \geq 0
\end{aligned}
$$

$$
\begin{aligned}
& \mathbf{y}^{T} c_{2} \leq 1+r_{f} \mathbf{y}^{T} \mathbf{1}<\mathbf{y}^{T} c_{3} \\
& \mathbf{y}^{T} \mathbf{1}-t \leq 0 \\
& y_{i} \geq 0, \quad i=1,2, \ldots, n \\
& t>0 .
\end{aligned}
$$

If $E\left[\mathbf{x}^{T} \xi\right] \geq \mathbf{x}^{T} c_{3}$, that is, $\mathbf{y}^{T} c_{3} \leq 1+r_{f} \mathbf{y}^{T} \mathbf{1}$, then we can solve model (33) by solving the following equivalent parametric programming:

$$
\min \frac{1}{2} \mathbf{y}^{T} C P C^{T} \mathbf{y}-\frac{\left[1-\lambda \theta_{r}+(1-\lambda) \theta_{l}\right]\left\{2 \mathbf{x}^{T}\left(c_{1}+c_{2}-3 c_{3}+c_{4}\right)-\left[\lambda \theta_{r}-(1-\lambda) \theta_{l}\right] \mathbf{x}^{T}\left(c_{1}-c_{2}-c_{3}+c_{4}\right)\right\}^{3}}{3072 \mathbf{x}^{T}\left(c_{4}-c_{3}\right)}
$$

subject to $\frac{\mathbf{y}^{T}\left(c_{1}+c_{2}+c_{3}+c_{4}\right)}{4}+\frac{\left[\lambda \theta_{r}-(1-\lambda) \theta_{l}\right] \mathbf{y}^{T}\left(c_{1}-c_{2}-c_{3}+c_{4}\right)}{8}-r_{f} \mathbf{y}^{T} \mathbf{1}-1 \geq 0$

$$
\begin{aligned}
& \mathbf{y}^{T} c_{3} \leq 1+r_{f} \mathbf{y}^{T} \mathbf{1} \\
& \mathbf{y}^{T} \mathbf{1}-t \leq 0 \\
& y_{i} \geq 0, \quad i=1,2, \ldots, n \\
& t>0 .
\end{aligned}
$$


TABLE 1: The parametric interval-valued trapezoidal fuzzy returns of twenty assets.

\begin{tabular}{lc}
\hline Asset $i$ & Parametric interval-valued fuzzy return \\
\hline 1 & {$\left[0.9946,0.9967,1.0012,1.0061 ; \theta_{l 1}, \theta_{r 1}\right]$} \\
2 & {$\left[0.9911,1.0002,1.0019,1.0052 ; \theta_{l 2}, \theta_{r 2}\right]$} \\
3 & {$\left[0.9966,1.0073,1.0081,1.0109 ; \theta_{l 3}, \theta_{r 3}\right]$} \\
4 & {$\left[0.9983,1.0090,1.0122,1.0135 ; \theta_{l 4}, \theta_{r 4}\right]$} \\
5 & {$\left[1.0059,1.0122,1.0262,1.0279 ; \theta_{l 5}, \theta_{r 5}\right]$} \\
6 & {$\left[1.0099,1.0159,1.0248,1.0429 ; \theta_{l 6}, \theta_{r 6}\right]$} \\
7 & {$\left[1.0209,1.0225,1.0416,1.0553 ; \theta_{l 7}, \theta_{r 7}\right]$} \\
8 & {$\left[1.0291,1.0299,1.0468,1.0678 ; \theta_{l 8}, \theta_{r 8}\right]$} \\
9 & {$\left[1.0201,1.0504,1.0651,1.0709 ; \theta_{l 9}, \theta_{r 9}\right]$} \\
10 & {$\left[1.0305,1.0399,1.0469,1.1093 ; \theta_{l 10}, \theta_{r 10}\right]$} \\
11 & {$\left[1.0304,1.0468,1.0712,1.0908 ; \theta_{l 11}, \theta_{r 11}\right]$} \\
12 & {$\left[1.0365,1.0529,1.0698,1.1096 ; \theta_{l 12}, \theta_{r 12}\right]$} \\
13 & {$\left[1.0214,1.0699,1.0778,1.1004 ; \theta_{l 13}, \theta_{r 13}\right]$} \\
14 & {$\left[1.0391,1.0699,1.0969,1.1096 ; \theta_{l 14}, \theta_{r 14}\right]$} \\
15 & {$\left[1.0422,1.0766,1.0977,1.1178 ; \theta_{l 15}, \theta_{r 15}\right]$} \\
16 & {$\left[1.0373,1.0914,1.0972,1.1171 ; \theta_{l 16}, \theta_{r 16}\right]$} \\
17 & {$\left[1.0604,1.0786,1.1183,1.1282 ; \theta_{l 17}, \theta_{r 17}\right]$} \\
18 & {$\left[1.0460,1.0952,1.1098,1.1361 ; \theta_{l 18}, \theta_{r 18}\right]$} \\
19 & {$\left[1.0415,1.0885,1.1155,1.1475 ; \theta_{l 19}, \theta_{r 19}\right]$} \\
20 & {$\left[1.0326,1.0896,1.1330,1.1392 ; \theta_{l 20}, \theta_{r 20}\right]$} \\
\hline &
\end{tabular}

Models (35)-(37) are three equivalent parametric submodels of model (33) in the different partitions of feasible region. For any given parameter values, based on the previous discussion, submodels (35) and (37) are determinate nonlinear programming. Meanwhile, submodel (36) with given parameters is a quadratic convex programming. They all can be solved by standard software.

\section{The Domain Decomposition Method}

The domain decomposition method (DDM) is a decomposition-based computation technique. It searches every partition of the feasible region to find a global optimum of the optimization problem. To begin with, the feasible region should be reasonably split into multiple subregions using the different cases existing in the original problem or different values of parameters. In each individual subregion, the subproblem corresponding to the original problem is not identical, and the differences between subproblems lie in the objective function or constraints. Next, to find the optimal solution of the original problem, it is necessary to design or select an appropriate algorithm and solve the subproblems according to the characteristics of the subproblems. Finally, we obtain the global optimum of original problem by comparing the obtained local optimal values of all subproblems.
To be specific, the solution process mainly comprises the following three steps.

Step 1. Solve three equivalent parametric submodels (35)(37), and find the local optimal solutions.

Step 2. Compute the objective values of three submodels (35)-(37) corresponding to these local optimal solutions, respectively.

Step 3. Compare the obtained optimal values of submodels (35)-(37), and choose the minimum as the global optimal value of original model (17).

As mentioned in Section 5.2, the feasible region of model (33) is decomposed into three disjoint subregions. Models (35)-(37) are the equivalent parametric subproblems of model (33) in the different subregions, and for any given parameters $\theta=\left(\theta_{l}, \theta_{r}\right)$ and $\lambda$ they just determinate nonlinear programming which can be solved by classic algorithms. Lingo is a comprehensive tool designed to build and solve linear, nonlinear (convex or nonconvex), quadratic, and integer optimization models. However, the ability to solve programming is also constrained by the number of variables. Therefore, it is necessary to develop a more targeted efficient algorithm to the proposed models. In this paper, we employ the DDM combined Lingo software to solve model (33). In the next section, we will demonstrate the validity and practicability of this method via numerical experiments.

\section{Numerical Example}

Consider an asset market which has twenty risk assets and a risk-free asset, and an investor wants to decide on an appropriate mix of assets as his/her investment portfolio. The risk-free asset is usually a treasury bond with the fixed return rate $r_{f}$. The short-selling is assumed to be prohibited for any assets in this market. Due to the impacts of the economic and political factors, the returns of these assets are uncertain, and their exact possibility distributions cannot be obtained. Thus, in this paper, these twenty risk assets' returns are presented by parametric interval-valued trapezoidal fuzzy variables $\tilde{\xi}_{i}=$ $\left[r_{1 i}, r_{2 i}, r_{3 i}, r_{4 i} ; \theta_{l i}, \theta_{r i}\right], i=1, \ldots, 20$ with variable possibility distributions, and the uncertain returns of twenty candidate assets are given in Table 1.

7.1. Computational Result under Parametric Interval-Valued Fuzzy Returns. Firstly, when the exact possibility distributions of asset returns are unavailable, we conduct the experiments under variable possibility distribution to find the optimal portfolio selection policy.

In our experiments, we set the values of parameters $\theta_{l i}$ and $\theta_{r i}, i=1,2, \ldots, 20$ as

$$
\begin{aligned}
\left(\theta_{l 1}, \theta_{l 2}, \ldots, \theta_{l 20}\right) & =(0.8,0.2,0.3,0.9,0.7,0.5,0.4,0.6,0.8,0.1,0.6,0.3,0.5,0.3,0.7,0.4,0.2,0.8,0.5,0.2) \\
\left(\theta_{r 1}, \theta_{r 2}, \ldots, \theta_{r 20}\right) & =(0.3,0.8,0.2,0.6,0.3,0.5,0.3,0.9,0.7,0.5,0.4,0.6,0.7,0.4,0.9,0.8,0.5,0.2,0.8,0.2)
\end{aligned}
$$


TABLE 2: The allocation proportions of model (33) with $\theta_{l}=0.9, \theta_{r}=0.2$, and $\lambda=0$ (\%).

\begin{tabular}{ccccccccc}
\hline$r_{f}$ & $x_{1}$ & $x_{3}$ & $x_{4}$ & $x_{8}$ & $x_{16}$ & $x_{17}$ & $x_{18}$ \\
\hline 0.0033 & 37.08982 & 51.43058 & 11.47960 & 0.000000 & 0.000000 & 0.000000 & 0.000000 & 0.000000 \\
0.0093 & 0.000000 & 53.50372 & 37.74321 & 8.753070 & 0.000000 & 0.000000 & 0.000000 & 0.000000 \\
0.0193 & 0.000000 & 47.97375 & 36.58445 & 0.000000 & 2.875511 & 12.56628 & 0.000000 & 0.000000 \\
0.0438 & 0.000000 & 27.74888 & 26.18283 & 0.000000 & 25.06405 & 21.00424 & 0.000000 & 0.000000 \\
0.0848 & 0.000000 & 0.000000 & 22.61346 & 0.000000 & 62.15479 & 35.32249 & 0.000000 & 0.000000 \\
0.0925 & 0.000000 & 0.000000 & 0.000000 & 0.000000 & 11.84791 & 13.55289 & 74.59920 & 0.000000 \\
0.0940 & 0.000000 & 0.000000 & 0.000000 & 0.000000 & 0.000000 & 3.144038 & 86.73522 & 10.12074 \\
0.0965 & 0.000000 & 0.000000 & 0.000000 & 0.000000 & 0.000000 & 0.000000 & 2.145002 & 97.85500 \\
0.0970 & - & - & - & - & - & - & - \\
\hline
\end{tabular}

TABLE 3: The allocation proportions of model (33) with $\theta_{l}=0.9, \theta_{r}=0.2$, and $\lambda=0.25$ (\%).

\begin{tabular}{lcccccccc}
\hline$r_{f}$ & $x_{1}$ & $x_{3}$ & $x_{4}$ & $x_{8}$ & $x_{16}$ & $x_{17}$ & $x_{18}$ \\
\hline 0.0033 & 38.99274 & 60.41775 & 0.000000 & 0.589515 & 0.000000 & 0.000000 & 0.000000 & 0.000000 \\
0.0093 & 5.941417 & 61.16051 & 22.99126 & 9.906809 & 0.000000 & 0.000000 & 0.000000 & 0.000000 \\
0.0193 & 0.000000 & 50.47323 & 34.22256 & 0.000000 & 3.955556 & 11.34866 & 0.000000 & 0.000000 \\
0.0438 & 0.000000 & 53.58670 & 0.000000 & 0.000000 & 26.61964 & 19.79366 & 0.000000 & 0.000000 \\
0.0848 & 0.000000 & 2.979972 & 0.000000 & 0.000000 & 65.03688 & 31.98315 & 0.000000 & 0.000000 \\
0.0925 & 0.000000 & 0.000000 & 0.000000 & 0.000000 & 17.58623 & 12.23930 & 70.17447 & 0.000000 \\
0.0940 & 0.000000 & 0.000000 & 0.000000 & 0.000000 & 3.478543 & 6.432623 & 90.08883 & 0.000000 \\
0.0965 & 0.000000 & 0.000000 & 0.000000 & 0.000000 & 0.000000 & 28.36709 & 7.005321 & 64.62759 \\
0.0970 & 0.000000 & 0.000000 & 0.000000 & 0.000000 & 0.000000 & 5.780347 & 0.000000 & 94.21965 \\
\hline
\end{tabular}

TABLE 4: The allocation proportions of model (33) with $\theta_{l}=0.9, \theta_{r}=0.2$, and $\lambda=0.5(\%)$.

\begin{tabular}{|c|c|c|c|c|c|c|c|c|}
\hline$r_{f}$ & $x_{1}$ & $x_{3}$ & $x_{4}$ & $x_{8}$ & $x_{16}$ & $x_{17}$ & $x_{18}$ & $x_{19}$ \\
\hline 0.0033 & 38.02403 & 61.84081 & 0.000000 & 0.135164 & 0.000000 & 0.000000 & 0.000000 & 0.000000 \\
\hline 0.0093 & 3.779654 & 85.59803 & 0.000000 & 10.62232 & 0.000000 & 0.000000 & 0.000000 & 0.000000 \\
\hline 0.0193 & 0.000000 & 84.02355 & 0.000000 & 0.000000 & 4.756157 & 11.22030 & 0.000000 & 0.000000 \\
\hline 0.0438 & 0.000000 & 53.91651 & 0.000000 & 0.000000 & 28.45683 & 17.62665 & 0.000000 & 0.000000 \\
\hline 0.0848 & 0.000000 & 3.533319 & 0.000000 & 0.000000 & 68.11919 & 28.34749 & 0.000000 & 0.000000 \\
\hline 0.0925 & 0.000000 & 0.000000 & 0.000000 & 0.000000 & 23.96391 & 10.77935 & 65.25674 & 0.000000 \\
\hline 0.0940 & 0.000000 & 0.000000 & 0.000000 & 0.000000 & 9.371306 & 5.083677 & 85.54502 & 0.000000 \\
\hline 0.0965 & 0.000000 & 0.000000 & 0.000000 & 0.000000 & 0.000000 & 69.14263 & 0.000000 & 30.85737 \\
\hline 0.0970 & 0.000000 & 0.000000 & 0.000000 & 0.000000 & 0.000000 & 37.53457 & 0.000000 & 62.46543 \\
\hline
\end{tabular}

Since $\theta_{l}=\max _{1 \leq i \leq n} \theta_{l i}$ and $\theta_{r}=\min _{1<i<n} \theta_{r i}$, we have $\theta_{l}=0.9$, $\theta_{r}=0.2$.

Assume the investor selects the values of $\lambda$ as $0,0.25$, $0.5,0.75$, and 1 . To test the effect of the risk-free return rate on the portfolio decision, we adjust the value of $r_{f}$ and do some experiments, and the returns of the risk-free asset are set as follows $r_{f}=0.33 \%, 0.93 \%, 1.93 \%, 4.38 \%, 8.48 \%, 9.25 \%$, $9.40 \%, 9.65 \%$, and $9.70 \%$. The obtained optimal allocation proportions to twenty risk assets are reported in Tables 2-6. Note that, according to $\mathbf{x}^{*}=\mathbf{y}^{*} / t^{*}$, we have the allocation proportions $x_{i}$ easily by reducing $y_{i}$ with the same ratio $t$. For simplicity, we give directly the results of $x_{i}$.

Next, we illustrate the solving process of DDM in detail with the case $r_{f}=0.0033$ and $\lambda=0$. To begin with, we solve the equivalent parametric subproblems (35)(37) with the fixed parameters values, respectively. By Lingo software, the objective value of subproblem (35) is $0.7501525 \times 10^{-6}$ with solution $\left(x_{1}, x_{3}, x_{4}\right)=(37.08982 \%$, $51.43058 \%, 11.47960 \%)$. In the meantime, after 21 solver iterations, the obtained objective value of subproblem (36) is $0.2788316 \times 10^{-5}$ with solution $\left(x_{1}, x_{3}, x_{8}\right)=$ (39.85010\%, 59.15827\%, 0.9916381\%). Moreover, the objective value and solution of subproblem (37) in this case are $0.1276898 \times 10^{-4}$ and $\left(x_{3}, x_{10}\right)=(81.86165 \%, 17.00404 \%)$. Obviously, $0.7501525 \times 10^{-6}$ is less than the other two objective values. According to DDM, the optimal solution of model (33) is the results of subproblem (35); that is, $x^{*}=$ $\left(x_{1}, x_{3}, x_{4}\right)=(37.08982 \%, 51.43058 \%, 11.47960 \%)$. Under 
TABLE 5: The allocation proportions of model (33) with $\theta_{l}=0.9, \theta_{r}=0.2$, and $\lambda=0.75$ (\%).

\begin{tabular}{|c|c|c|c|c|c|c|c|c|}
\hline$r_{f}$ & $x_{1}$ & $x_{3}$ & $x_{4}$ & $x_{8}$ & $x_{16}$ & $x_{17}$ & $x_{18}$ & $x_{19}$ \\
\hline 0.0033 & 37.73585 & 62.26415 & 0.000000 & 0.000000 & 0.000000 & 0.000000 & 0.000000 & 0.000000 \\
\hline 0.0093 & 2.467786 & 87.52520 & 0.000000 & 10.00701 & 0.000000 & 0.000000 & 0.000000 & 0.000000 \\
\hline 0.0193 & 0.000000 & 54.51039 & 0.000000 & 42.69392 & 2.795687 & 0.000000 & 0.000000 & 0.000000 \\
\hline 0.0438 & 0.000000 & 3.033993 & 0.000000 & 73.24945 & 23.71656 & 0.000000 & 0.000000 & 0.000000 \\
\hline 0.0848 & 0.000000 & 0.000000 & 0.000000 & 6.626968 & 73.65104 & 19.72199 & 0.000000 & 0.000000 \\
\hline 0.0925 & 0.000000 & 0.000000 & 0.000000 & 0.000000 & 31.09411 & 9.147131 & 59.75876 & 0.000000 \\
\hline 0.0940 & 0.000000 & 0.000000 & 0.000000 & 0.000000 & 15.95938 & 3.575565 & 80.46506 & 0.000000 \\
\hline 0.0965 & 0.000000 & 0.000000 & 0.000000 & 0.000000 & 0.000000 & 88.80842 & 0.000000 & 11.19158 \\
\hline 0.0970 & 0.000000 & 0.000000 & 0.000000 & 0.000000 & 0.000000 & 61.21745 & 0.000000 & 38.78255 \\
\hline
\end{tabular}

TABLE 6: The allocation proportions of model (33) with $\theta_{l}=0.9, \theta_{r}=0.2$, and $\lambda=1(\%)$.

\begin{tabular}{|c|c|c|c|c|c|c|c|c|}
\hline$r_{f}$ & $x_{1}$ & $x_{3}$ & $x_{4}$ & $x_{8}$ & $x_{16}$ & $x_{17}$ & $x_{18}$ & $x_{19}$ \\
\hline 0.0033 & 37.73585 & 62.26415 & 0.000000 & 0.000000 & 0.000000 & 0.000000 & 0.000000 & 0.000000 \\
\hline 0.0093 & 0.960099 & 89.74003 & 0.000000 & 9.299870 & 0.000000 & 0.000000 & 0.000000 & 0.000000 \\
\hline 0.0193 & 0.000000 & 46.90265 & 0.000000 & 53.09735 & 0.000000 & 0.000000 & 0.000000 & 0.000000 \\
\hline 0.0438 & 0.000000 & 0.000000 & 0.000000 & 77.39837 & 22.60163 & 0.000000 & 0.000000 & 0.000000 \\
\hline 0.0848 & 0.000000 & 0.000000 & 0.000000 & 0.000000 & 48.43750 & 51.56250 & 0.000000 & 0.000000 \\
\hline 0.0925 & 0.000000 & 0.000000 & 0.000000 & 0.000000 & 39.11838 & 7.310251 & 53.57137 & 0.000000 \\
\hline 0.0940 & 0.000000 & 0.000000 & 0.000000 & 0.000000 & 23.37353 & 1.878349 & 74.74812 & 0.000000 \\
\hline 0.0965 & 0.000000 & 0.000000 & 0.000000 & 0.153667 & 0.000000 & 99.84633 & 0.000000 & 0.000000 \\
\hline 0.0970 & 0.000000 & 0.000000 & 0.000000 & 0.000000 & 0.000000 & 79.55936 & 0.000000 & 20.44064 \\
\hline
\end{tabular}

TABLE 7: The obtained optimal portfolio results by comparing three submodels when $\theta_{l}=0.9, \theta_{r}=0.2$, and $\lambda=0$.

\begin{tabular}{lccccccccc}
\hline$r_{f}$ & 0.0033 & 0.0093 & 0.0193 & 0.0438 & 0.0848 & 0.0925 & 0.0940 & 0.0965 & 0.0970 \\
\hline Model (35) & $*$ & $*$ & $*$ & $*$ & $*$ & $*$ & $*$ & - & - \\
Model (36) & $\sqrt{ }$ & $\sqrt{ }$ & $\sqrt{ }$ & $\sqrt{ }$ & $\sqrt{ }$ & $\sqrt{ }$ & $\sqrt{ }$ & $*$ & - \\
Model (37) & $\sqrt{ }$ & $\sqrt{ }$ & $\sqrt{ }$ & $\sqrt{ }$ & - & - & - & - \\
\hline
\end{tabular}

Notations: $*$ : optimal; $\sqrt{ }$ : feasible but nonoptimal; -: unfeasible.

the different values of $r_{f}$, how to find the optimal portfolio through comparing three submodels determined by DDM are shown in Table 7.

7.2. Computational Result under Fuzzy Returns. For the sake of comparison, in this subsection, we solve model (33) with fuzzy returns. The fuzzy returns are characterized by the principle possibility distributions of interval-valued fuzzy variables, which are obtained by setting $\theta_{l j}=\theta_{r j}=0$ in parametric possibility distributions. Then, the returns of twenty securities are trapezoidal fuzzy variables $\xi_{j}^{p}=\left(r_{1 j}\right.$, $\left.r_{2 j}, r_{3 j}, r_{4 j}\right), j=1,2, \ldots, 20$, and their expected values and semimoments are given in Table 8 .

Employing Lingo software, we solve model (33) and obtain the optimal allocation policy in Table 9. The results imply that the investor should put the money to different assets under different risk-free return levels.

7.3. Parameter Analysis and Results Comparison. In this subsection, we will analyze the parameters in the fuzzy PSR portfolio selection model. In order to identify the parameter's influence on the optimal portfolio selection, we solved the model with different values of parameters.

Case 1 (the influence of parameter $\theta$ ). The parameter $\theta=$ $\left(\theta_{l}, \theta_{r}\right)$ characterizes the degree of uncertainty of a parametric interval-valued fuzzy return. For example, regarding the parametric interval-valued fuzzy variable $\left[1,2,3,4 ; \theta_{l}, \theta_{r}\right]$, the changeable range of parametric possibility distributions with different parameters $\theta=\left(\theta_{l}, \theta_{r}\right)$ is shown in Figure 1 . From the figure, we know that the value of parameter $\theta$ is bigger; the uncertainty degree of fuzzy return is higher.

We have computed the model with two sets of values: $\theta_{l}=$ $0.9, \theta_{r}=0.2$, and $\theta_{l}=\theta_{r}=0$ in Tables 2 and 9. These results show that the optimal allocation policies are different with the variation of parameter $\theta$. For example, if $r_{f}=0.0033$, when $\theta_{l}=0.9, \theta_{r}=0.2$, and $\lambda=0$, the optimal allocation policy is selecting three assets with numbers 1,3 , and 4 . In the meantime, if $r_{f}=0.0033$, when $\theta_{l}=\theta_{r}=0$, the investor should choose two assets with numbers 1 and 3 . The allocation proportions are distinctly different. 
TABLE 8: The values of $E\left[\xi_{j}^{p}\right]$ and $\operatorname{SM}\left[\xi_{j}^{p}\right]$ of twenty fuzzy returns.

\begin{tabular}{llll}
\hline Asset & Fuzzy return $\left(r_{1 j}, r_{2 j}, r_{3 j}, r_{4 j}\right)$ & $E\left[\xi_{j}^{p}\right]$ & $\mathrm{SM}\left[\xi_{j}^{p}\right]$ \\
\hline 1 & $\xi_{1}^{p}=(0.9946,0.9967,1.0012,1.0061)$ & 0.999650 & 0.999600 \\
2 & $\xi_{2}^{p}=(0.9911,1.0002,1.0019,1.0052)$ & 1.005725 & $1.12477 \times 10^{-5}$ \\
3 & $\xi_{3}^{p}=(0.9966,1.0073,1.0081,1.0109)$ & 1.008250 & $1.18349 \times 10^{-5}$ \\
4 & $\xi_{4}^{p}=(0.9983,1.0090,1.0122,1.0135)$ & 1.018050 & $1.53438 \times 10^{-5}$ \\
5 & $\xi_{5}^{p}=(1.0059,1.0122,1.0262,1.0279)$ & 1.023375 & $4.21537 \times 10^{-5}$ \\
6 & $\xi_{6}^{p}=(1.0099,1.0159,1.0248,1.0429)$ & 1.035075 & $5.63628 \times 10^{-5}$ \\
7 & $\xi_{7}^{p}=(1.0209,1.0225,1.0416,1.0553)$ & 1.043400 & $8.95520 \times 10^{-5}$ \\
8 & $\xi_{8}^{p}=(1.0291,1.0299,1.0468,1.0678)$ & 1.051625 & $9.66317 \times 10^{-5}$ \\
9 & $\xi_{9}^{p}=(1.0201,1.0504,1.0651,1.0709)$ & 1.056650 & 1.059800 \\
10 & $\xi_{10}^{p}=(1.0305,1.0399,1.0469,1.1093)$ & 1.067200 & $2.72324 \times 10^{-4}$ \\
11 & $\xi_{11}^{p}=(1.0304,1.0468,1.0712,1.0908)$ & 1.067375 & $2.35927 \times 10^{-4}$ \\
12 & $\xi_{12}^{p}=(1.0365,1.0529,1.0698,1.1096)$ & 1.078875 & $3.33943 \times 10^{-4}$ \\
13 & $\xi_{13}^{p}=(1.0214,1.0699,1.0778,1.1004)$ & 1.083575 & $3.36597 \times 10^{-4}$ \\
14 & $\xi_{14}^{p}=(1.0391,1.0699,1.0969,1.1096)$ & 1.085750 & $3.41522 \times 10^{-4}$ \\
15 & $\xi_{15}^{p}=(1.0422,1.0766,1.0977,1.1178)$ & 1.096375 & $3.50375 \times 10^{-4}$ \\
16 & $\xi_{16}^{p}=(1.0373,1.0914,1.0972,1.1171)$ & 1.096775 & $3.74934 \times 10^{-4}$ \\
17 & $\xi_{17}^{p}=(1.0604,1.0786,1.1183,1.1282)$ & 1.098250 & $4.43425 \times 10^{-4}$ \\
18 & $\xi_{18}^{p}=(1.0460,1.0952,1.1098,1.1361)$ & 1.098600 & $6.44823 \times 10^{-2}$ \\
19 & $\xi_{19}^{p}=(1.0415,1.0885,1.1155,1.1475)$ & & $8.38500 \times 10^{-2}$ \\
20 & $\xi_{20}^{p}=(1.0326,1.0896,1.1330,1.1392)$ &
\end{tabular}

TABLE 9: The allocation proportions of model (33) with $\theta_{l}=0, \theta_{r}=0(\%)$.

\begin{tabular}{lccccccc}
\hline$r_{f}$ & $x_{1}$ & $x_{3}$ & $x_{8}$ & $x_{16}$ & $x_{17}$ & $x_{18}$ \\
\hline 0.0033 & 37.73585 & 62.26415 & 0.000000 & 0.000000 & 0.000000 & 0.000000 & 0.000000 \\
0.0093 & 2.077768 & 88.09815 & 9.824086 & 0.000000 & 0.000000 & 0.000000 & 0.000000 \\
0.0193 & 0.000000 & 46.90265 & 53.09735 & 0.000000 & 0.000000 & 0.000000 & 0.000000 \\
0.0438 & 0.000000 & 0.000000 & 77.39837 & 22.60163 & 0.000000 & 0.000000 & 0.000000 \\
0.0848 & 0.000000 & 0.000000 & 6.882793 & 74.62438 & 18.49283 & 0.000000 & 0.000000 \\
0.0925 & 0.000000 & 0.000000 & 0.000000 & 33.18711 & 8.668010 & 58.14488 & 0.000000 \\
0.0940 & 0.000000 & 0.000000 & 0.000000 & 17.89324 & 3.132873 & 78.97389 & 0.000000 \\
0.0965 & 0.000000 & 0.000000 & 0.000000 & 0.000000 & 93.33333 & 0.000000 & 6.666667 \\
0.0970 & 0.000000 & 0.000000 & 0.000000 & 0.000000 & 66.66667 & 0.000000 & 33.33333 \\
\hline
\end{tabular}

Case 2 (the influence of parameter $\lambda$ ). The parameter $\lambda$ is a real number in $[0,1]$, and it determines the location of variable possibility distribution. The $\lambda$ selection is a variable representative of a parametric interval-valued fuzzy variable. Given the parameter $\theta$, the variation of $\lambda$ can make the possibility distribution run over the entire support of a parametric interval-valued fuzzy variable. Figure 2 shows the parametric possibility distributions of five selection variables of parametric interval-valued fuzzy variable $[0,2,3,5 ; 0.9,0.2]$ with parameter $\lambda=0,0.25,0.5,0.75,1$.

Assume parameters $\theta_{l}=0.9, \theta_{r}=0.2$; Tables $2-6$ give the results of asset allocation with different $\lambda$. And we know that the selected assets of optimal portfolio vary in the candidate assets, and allocation proportions are different. From the results of the optimal value, we observe that the value of parameter $\lambda$ is bigger; the portfolio risk is smaller.
So for the proposed model we can reduce the portfolio risk by choosing the bigger value of parameter $\lambda$. Thus we draw the conclusion that the model is sensitive to parameter, and the portfolio risk is negative correlation with parameter $\lambda$.

7.4. Discussion. In this subsection, we will carry out a discussion about the difference between the variable possibility distribution and fixed possibility distribution. From the computational results in Sections 7.1 and 7.2, we obtain the following findings.

Firstly, when the fixed possibility distributions of uncertain returns are available, the portfolio selection model is a fuzzy optimization model. In this case, the optimal allocation policies are listed in Table 9. From the computational results, we can observe that when the return level of the risk-free asset $r_{f}$ increases, the optimal portfolio proportions are 


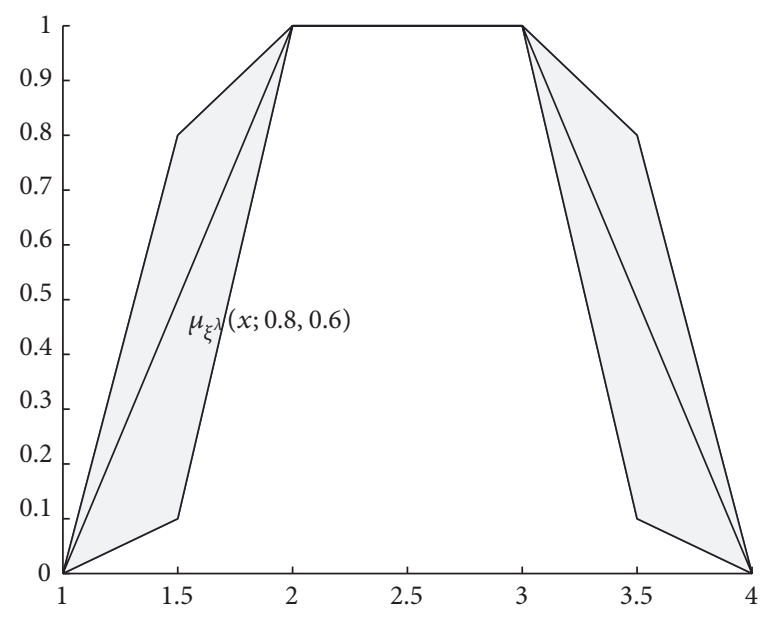

(a) $\theta_{l}=0.8, \theta_{r}=0.6$

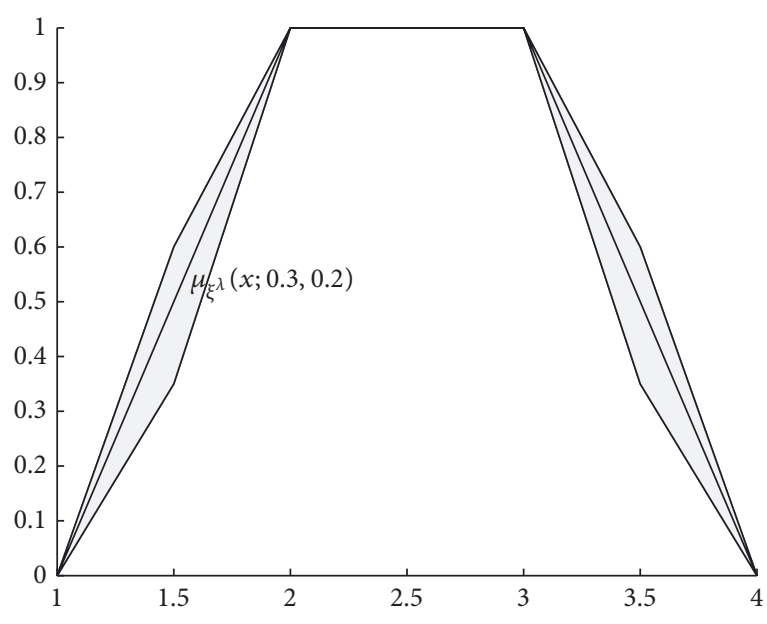

(b) $\theta_{l}=0.3, \theta_{r}=0.2$

FIGURE 1: The changeable range of parametric possibility distribution of parametric interval-valued fuzzy variable $\left[1,2,3,4 ; \theta_{l}, \theta_{r}\right]$.

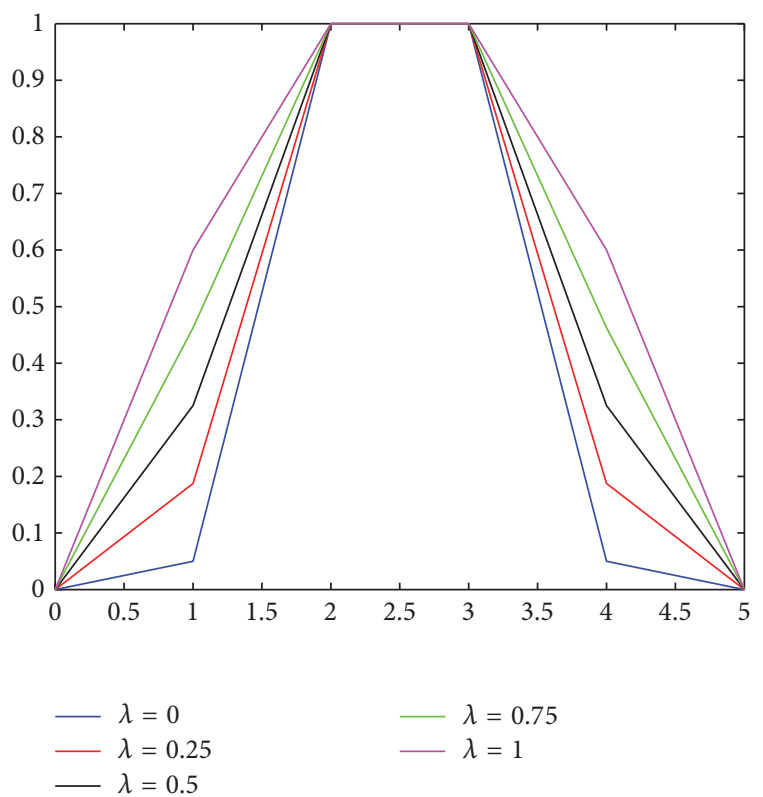

Figure 2: The possibility distributions of $[0,2,3,5 ; 0.9,0.2]$ with five different values of $\lambda$.

different. For example, when $r_{f}$ is 0.0093 , the investor should allocate his/her money to three assets 1,3 , and 8; when $r_{f}$ is 0.0848 , the investor should select assets 8,16 , and 17 . It is worth noting that if the decision maker cannot obtain the precise distribution information before making decisions, this method will be invalid.

Secondly, when the fixed possibility distributions cannot be obtained during the portfolio selection process, we can characterize the uncertain returns with parametric intervalvalued fuzzy distributions. In this situation, the portfolio selection model is a fuzzy parametric optimization model, in which two types of parameters are embedded. From the computational results in Tables $2-6$, we can see that the selected assets and allocation proportions change with the variation of parameter $\lambda$ in $[0,1]$. Specifically, when the return level of the risk-free asset $r_{f}$ is 0.0193 , if $\lambda=0$ and 0.25 , the decision maker should allocate his/her money to four assets with numbers $3,4,16$, and 17 , while if $\lambda=0.5$ and 0.75 , the money is allocated to three assets; and if $\lambda=1$, the selected assets are only two ones with numbers 3 and 8 . Obviously, the optimal portfolio policy is closely related to parameter $\lambda$. Furthermore, comparing the variable possibility distribution method with the fixed possibility distribution method, we know the two methods can provide different investment while the return level $r_{f}$ varies from 0.0033 to 0.0970. Even though they provide diversified investment for the same assets, the allocation proportions to them are different.

Finally, in other methods of interval fuzzy optimization, the fixed lower/upper bounds or midpoint of the distributional interval is often chosen as representative of the family of possibility distributions. In our parametric interval-valued method, the possibility distribution of $\lambda$ selection variable runs over the entire fuzzy support as the parameter $\lambda$ varies in the unit interval $[0,1]$. Namely, the variable possibility distribution of selection variable can be any distribution in the fuzzy support. Thus the parametric optimization method based on the variable possibility distribution is flexible and comprehensive for decision makers. Particularly, if the investor cannot obtain the exact distributional information, the method of variable possibility distribution provides an effectual way for decision makers to make their informed portfolio selection policies.

\section{Conclusions}

In portfolio management, an important role of performance measure is to help investors evaluate their portfolios and make a portfolio selection decision. This paper presents a theoretically sound portfolio performance to optimize the 
investment policy, and the main conclusions are summarized as follows:

(i) A new downside risk measure: the semimoment of the selection variable is presented to characterize the loss risk. Using the proposed measure, we modify the conventional Sharpe ratio and define a parametric Sharpe ratio for fuzzy portfolio performance evaluation.

(ii) A novel fuzzy PSR portfolio selection model is built wherein the uncertain returns are characterized by interval-valued possibility distributions. When the exact distributions of uncertain returns are difficult to be determined in advance, the proposed approach could be employed to characterize the uncertainty in practice.

(iii) By performing an equivalent transformation, the proposed model is transformed into nonfractional programming. Furthermore, according to structure characteristics, a domain decomposition method is introduced and the proposed model is solved by computing three equivalent parametric submodels.

(iv) We conduct the experiments and compare the computational results under variable possibility distribution with fixed possibility distribution and analyze the influence of different parameters on the optimal portfolio.

Future academical innovation directions might address the following two topics. On the one hand, the current risk measure used in this paper is semimoment; an extension to other downside risk measure is possible. On the other hand, the present model finds the optimal portfolio decision via parametric optimization method; the case dealing with distributionally robust optimization method based on the uncertain distribution set is a future avenue for our research.

\section{Appendix}

Proof of Theorem 2. We only prove assertion (i); the other assertions can be proved similarly.
Let $\widetilde{\xi}_{i}=\left[r_{1 i}, r_{2 i}, r_{3 i}, r_{4 i} ; \theta_{l i}, \theta_{r i}\right]$ be parametric intervalvalued trapezoidal fuzzy variable. Since the principle possibility distributions of $\widetilde{\xi}_{i}$ are mutually independent in the sense of [38], their linear combination $\widetilde{\xi}=\mathbf{x}^{T} \widetilde{\xi}$ is a parametric interval-valued trapezoidal fuzzy variable [32] and denoted by $\left[r_{1}, r_{2}, r_{3}, r_{4} ; \theta_{l}, \theta_{r}\right]$, where

$$
\begin{aligned}
& r_{1}=\sum_{i=1}^{n} x_{i} r_{1 i}, \\
& r_{2}=\sum_{i=1}^{n} x_{i} r_{2 i}, \\
& r_{3}=\sum_{i=1}^{n} x_{i} r_{3 i}, \\
& r_{4}=\sum_{i=1}^{n} x_{i} r_{4 i},
\end{aligned}
$$

with $\theta_{l}=\max _{1 \leq i \leq n} \theta_{l i}$ and $\theta_{r}=\min _{1 \leq i \leq n} \theta_{r i}$. For simplicity, these parameters are also expressed as

$$
\begin{aligned}
& r_{1}=\mathbf{x}^{T} c_{1}, \\
& r_{2}=\mathbf{x}^{T} c_{2}, \\
& r_{3}=\mathbf{x}^{T} c_{3}, \\
& r_{4}=\mathbf{x}^{T} c_{4} .
\end{aligned}
$$

For $\tilde{\xi}=\mathbf{x}^{T} \widetilde{\xi}$ is a parametric interval-valued trapezoidal fuzzy variable $\left[r_{1}, r_{2}, r_{3}, r_{4} ; \theta_{l}, \theta_{r}\right]$ and $\boldsymbol{\xi}=\mathbf{x}^{T} \xi$ is its $\lambda$ selection, then the parametric possibility distribution of $\boldsymbol{\xi}$ is the following piecewise linear function:

$$
\mu_{\xi}(r ; \lambda, \theta)= \begin{cases}\frac{\left[1+\lambda \theta_{r}-(1-\lambda) \theta_{l}\right]\left(r-r_{1}\right)}{r_{2}-r_{1}}, & r_{1} \leq r \leq \frac{r_{1}+r_{2}}{2} \\ \frac{\left[1-\lambda \theta_{r}+(1-\lambda) \theta_{l}\right] r+\left[\lambda \theta_{r}-(1-\lambda) \theta_{l}\right] r_{2}-r_{1}}{r_{2}-r_{1}}, & \frac{r_{1}+r_{2}}{2}<r \leq r_{2} \\ 1, & r_{2}<r \leq r_{3} \\ \frac{\left[\lambda \theta_{r}-(1-\lambda) \theta_{l}-1\right] r+\left[(1-\lambda) \theta_{l}-\lambda \theta_{r}\right] r_{3}+r_{4}}{r_{4}-r_{3}}, & r_{3}<r \leq \frac{r_{3}+r_{4}}{2} \\ \frac{\left[1+\lambda \theta_{r}-(1-\lambda) \theta_{l}\right]\left(r_{4}-r\right)}{r_{4}-r_{3}}, & \frac{r_{3}+r_{4}}{2}<r \leq r_{4},\end{cases}
$$

where $\theta=\left(\theta_{l}, \theta_{r}\right)$.
From the expression of $\mu_{\xi}(r ; \lambda, \theta)$, the credibility distribution function of $\xi$ is the following nondecreasing function: 


$$
\operatorname{Cr}\{\boldsymbol{\xi} \leq r\}= \begin{cases}0, & r \leq r_{1} \\ \frac{\left[1+\lambda \theta_{r}-(1-\lambda) \theta_{l}\right]\left(r-r_{1}\right)}{2\left(r_{2}-r_{1}\right)}, & r_{1}<r \leq \frac{r_{1}+r_{2}}{2} \\ \frac{\left[1-\lambda \theta_{r}+(1-\lambda) \theta_{l}\right] r+\left[\lambda \theta_{r}-(1-\lambda) \theta_{l}\right] r_{2}-r_{1}}{2\left(r_{2}-r_{1}\right)}, & \frac{r_{1}+r_{2}}{2}<r \leq r_{2} \\ \frac{1}{2}, & r_{2}<r \leq r_{3} \\ \frac{\left[1-\lambda \theta_{r}+(1-\lambda) \theta_{l}\right] r+\left[\lambda \theta_{r}-(1-\lambda) \theta_{l}-2\right] r_{3}+r_{4}}{2\left(r_{4}-r_{3}\right)}, & r_{3}<r \leq \frac{r_{3}+r_{4}}{2} \\ \frac{\left[1-\lambda \theta_{r}+(1-\lambda) \theta_{l}\right] r_{4}+\left[1+\lambda \theta_{r}-(1-\lambda) \theta_{l}\right] r-2 r_{3}}{2\left(r_{2}-r_{1}\right)}, & \frac{r_{3}+r_{4}}{2}<r \leq r_{4} \\ 1, & r \geq r_{4} .\end{cases}
$$

If $m<r_{2}$, then the semimoment of $\boldsymbol{\xi}$ is computed by the following integral:

$$
\begin{aligned}
& \operatorname{SM}[\boldsymbol{\xi}]=\int_{(-\infty, m)}(m-r)^{2} \mathrm{~d}(\operatorname{Cr}\{\boldsymbol{\xi} \leq r\}) \\
& =\int_{\left(r_{1},\left(r_{1}+r_{2}\right) / 2\right)}(m-r)^{2} \mathrm{~d}\left(\frac{\left[1+\lambda \theta_{r}-(1-\lambda) \theta_{l}\right]\left(r-r_{1}\right)}{2\left(r_{2}-r_{1}\right)}\right) \\
& +\int_{\left(\left(r_{1}+r_{2}\right) / 2, m\right)}(m-r)^{2} \mathrm{~d}\left(\frac{\left[1-\lambda \theta_{r}+(1-\lambda) \theta_{l}\right] r+\left[\lambda \theta_{r}-(1-\lambda) \theta_{l}\right] r_{2}-r_{1}}{2\left(r_{2}-r_{1}\right)}\right) \\
& =\left[\lambda \theta_{r}-(1-\lambda) \theta_{l}\right]^{3} \frac{\left(r_{1}^{2}-2 r_{1} r_{2}+r_{2}^{2}-2 r_{1} r_{3}+2 r_{1} r_{4}+2 r_{2} r_{3}-2 r_{2} r_{4}-2 r_{3} r_{4}+r_{3}^{2}+r_{4}^{2}\right)}{256} \\
& +\left[\lambda \theta_{r}-(1-\lambda) \theta_{l}\right]^{2} \frac{\left(-7 r_{1}^{2}+6 r_{1} r_{2}+r_{2}^{2}+10 r_{1} r_{3}-2 r_{1} r_{4}+14 r_{2} r_{3}-6 r_{2} r_{4}-2 r_{3} r_{4}-3 r_{3}^{2}+5 r_{4}^{2}\right)}{256} \\
& +\left[\lambda \theta_{r}-(1-\lambda) \theta_{l}\right] \frac{\left(7 r_{1}^{2}+4 r_{1} r_{2}+r_{2}^{2}-3 r_{1} r_{3}-15 r_{1} r_{4}+9 r_{2} r_{3}-3 r_{2} r_{4}+6 r_{3} r_{4}+6 r_{4}^{2}\right)}{192} \\
& +\frac{\left(13 r_{1}^{2}-2 r_{1} r_{2}+r_{2}^{2}-12 r_{1} r_{3}-12 r_{1} r_{4}+9 r_{2} r_{3}-3 r_{2} r_{4}+6 r_{3} r_{4}+3 r_{3}^{2}+3 r_{4}^{2}\right)}{192} \\
& +\frac{\left[1-\lambda \theta_{r}+(1-\lambda) \theta_{l}\right]\left\{2\left(r_{1}+r_{2}-r_{3}-r_{4}\right)\right\}^{3}}{3072\left(r_{2}-r_{1}\right)} \text {. }
\end{aligned}
$$

It follows that

$$
\operatorname{SM}[\boldsymbol{\xi}]=\operatorname{SM}\left[\mathbf{x}^{T} \xi\right]=\frac{1}{2} r^{T} Q r-\frac{\left[1-\lambda \theta_{r}+(1-\lambda) \theta_{l}\right]\left\{2\left(r_{1}+r_{2}-r_{3}-r_{4}\right)-\left[\lambda \theta_{r}-(1-\lambda) \theta_{l}\right]\left(r_{1}-r_{2}-r_{3}+r_{4}\right)\right\}^{3}}{3072\left(r_{2}-r_{1}\right)}
$$


where $r=\left(r_{1}, r_{2}, r_{3}, r_{4}\right)^{T}=\left(\mathbf{x}^{T} c_{1}, \mathbf{x}^{T} c_{2}, \mathbf{x}^{T} c_{3}, \mathbf{x}^{T} c_{4}\right)^{T}$, and the elements of the symmetric matrix $Q$ include

$$
\begin{aligned}
& Q_{11}=\frac{1}{128}\left[\lambda \theta_{r}-(1-\lambda) \theta_{l}\right]^{3} \\
& -\frac{7}{128}\left[\lambda \theta_{r}-(1-\lambda) \theta_{l}\right]^{2} \\
& +\frac{7}{96}\left[\lambda \theta_{r}-(1-\lambda) \theta_{l}\right]+\frac{13}{96}, \\
& Q_{12}=-\frac{1}{32}\left[\lambda \theta_{r}-(1-\lambda) \theta_{l}\right]^{3} \\
& +\frac{3}{32}\left[\lambda \theta_{r}-(1-\lambda) \theta_{l}\right]^{2} \\
& +\frac{1}{12}\left[\lambda \theta_{r}-(1-\lambda) \theta_{l}\right]+\frac{1}{24}, \\
& Q_{13}=-\frac{1}{32}\left[\lambda \theta_{r}-(1-\lambda) \theta_{l}\right]^{3} \\
& +\frac{5}{32}\left[\lambda \theta_{r}-(1-\lambda) \theta_{l}\right]^{2} \\
& -\frac{1}{16}\left[\lambda \theta_{r}-(1-\lambda) \theta_{l}\right]+\frac{1}{4}, \\
& Q_{14}=\frac{1}{32}\left[\lambda \theta_{r}-(1-\lambda) \theta_{l}\right]^{3} \\
& -\frac{1}{32}\left[\lambda \theta_{r}-(1-\lambda) \theta_{l}\right]^{2} \\
& -\frac{5}{16}\left[\lambda \theta_{r}-(1-\lambda) \theta_{l}\right]-\frac{1}{4}, \\
& Q_{22}=\frac{1}{128}\left[\lambda \theta_{r}-(1-\lambda) \theta_{l}\right]^{3} \\
& +\frac{1}{128}\left[\lambda \theta_{r}-(1-\lambda) \theta_{l}\right]^{2} \\
& +\frac{1}{96}\left[\lambda \theta_{r}-(1-\lambda) \theta_{l}\right]+\frac{1}{96} \\
& Q_{23}=\frac{1}{32}\left[\lambda \theta_{r}-(1-\lambda) \theta_{l}\right]^{3} \\
& +\frac{7}{32}\left[\lambda \theta_{r}-(1-\lambda) \theta_{l}\right]^{2} \\
& +\frac{3}{16}\left[\lambda \theta_{r}-(1-\lambda) \theta_{l}\right], \\
& Q_{24}=-\frac{1}{32}\left[\lambda \theta_{r}-(1-\lambda) \theta_{l}\right]^{3} \\
& -\frac{3}{32}\left[\lambda \theta_{r}-(1-\lambda) \theta_{l}\right]^{2} \\
& -\frac{1}{16}\left[\lambda \theta_{r}-(1-\lambda) \theta_{l}\right], \\
& Q_{33}=\frac{1}{128}\left[\lambda \theta_{r}-(1-\lambda) \theta_{l}\right]^{3}
\end{aligned}
$$

$$
\begin{aligned}
& -\frac{3}{128}\left[\lambda \theta_{r}-(1-\lambda) \theta_{l}\right]^{2}+\frac{1}{32}, \\
Q_{34}= & -\frac{1}{32}\left[\lambda \theta_{r}-(1-\lambda) \theta_{l}\right]^{3} \\
& -\frac{1}{32}\left[\lambda \theta_{r}-(1-\lambda) \theta_{l}\right]^{2} \\
& +\frac{1}{8}\left[\lambda \theta_{r}-(1-\lambda) \theta_{l}\right]+\frac{1}{8}, \\
Q_{44}= & \frac{1}{128}\left[\lambda \theta_{r}-(1-\lambda) \theta_{l}\right]^{3} \\
& +\frac{5}{128}\left[\lambda \theta_{r}-(1-\lambda) \theta_{l}\right]^{2} \\
& +\frac{1}{16}\left[\lambda \theta_{r}-(1-\lambda) \theta_{l}\right]+\frac{1}{32} .
\end{aligned}
$$

Moreover, it is easy to check that the matrix $P$ of case (ii) is positive semidefinite, so the semimoment $\operatorname{SM}\left[\mathbf{x}^{T} \xi\right]$ in case (ii) is a parametric quadratic convex function with respect to decision vector $x \in R^{n}$. The proof of theorem is complete.

Proof of Corollary 3. The proof is similar to that of Theorem 2.

\section{Competing Interests}

The authors declare that there is no conflict of interests regarding the publication of this paper.

\section{Acknowledgments}

This work is supported by the Natural Science Foundation of China under Grant no. 61374184, the Natural Science Foundation of Hebei Province no. A2014201166, and PostGraduate's Innovation Fund Project of Hebei Province no. S2016017.

\section{References}

[1] H. Markowitz, "Portfolio selection," The Journal of Finance, vol. 7, no. 1, pp. 77-91, 1952.

[2] P. N. Kolm, R. Tütüncü, and F. J. Fabozzi, "60 Years of portfolio optimization: practical challenges and current trends," European Journal of Operational Research, vol. 234, no. 2, pp. 356-371, 2014.

[3] V. DeMiguel, L. Garlappi, F. J. Nogales, and R. Uppal, "A generalized approach to portfolio optimization: improving performance by constraining portfolio norms," Management Science, vol. 55, no. 5, pp. 798-812, 2009.

[4] S. Zhu and M. Fukushima, "Worst-case conditional value-atrisk with application to robust portfolio management," Operations Research, vol. 57, no. 5, pp. 1155-1168, 2009.

[5] H. Yao, Z. Li, and Y. Lai, "Mean-CVaR portfolio selection: a nonparametric estimation framework," Computers \& Operations Research, vol. 40, no. 4, pp. 1014-1022, 2013.

[6] F. He and R. Qu, "A two-stage stochastic mixed-integer program modelling and hybrid solution approach to portfolio selection 
problems," Information Sciences, vol. 289, no. 1, pp. 190-205, 2014.

[7] Y. Chen and Y. Wang, "Two-stage fuzzy portfolio selection problem with transaction costs," Mathematical Problems in Engineering, vol. 2015, Article ID 675157, 12 pages, 2015.

[8] W. F. Sharpe, "Mutual Fund Performance," The Journal of Business, vol. 39, no. 1, pp. 119-138, 1966.

[9] W. F. Sharpe, “The sharpe ratio," The Journal of Portfolio Management, vol. 21, no. 1, pp. 49-58, 1994.

[10] V. S. Bawa, "Optimal rules for ordering uncertain prospects," Journal of Financial Economics, vol. 2, no. 1, pp. 95-121, 1975.

[11] P. C. Fishburn, "Mean-risk analysis with risk associated with below-target returns," American Economic Review, vol. 67, no. 2, pp. 116-126, 1977.

[12] F. A. Sortino and R. van der Meer, "Downside risk," The Journal of Portfolio Management, vol. 17, no. 4, pp. 27-31, 1991.

[13] C. Keating and W. F. Shadwick, "A universal performance measure," Journal of Performance Measurement, vol. 6, no. 3, pp. 59-84, 2002.

[14] P. D. Kaplan and J. A. Knowles, "Kappa: a generalized downside risk-adjusted performance measure," Journal of Performance Measurement, vol. 8, no. 3, pp. 42-54, 2004.

[15] L. Favre and J. Galeano, "Mean-modified value-at-risk optimization with hedge funds," The Journal of Alternative Investments, vol. 5, no. 2, pp. 21-25, 2002.

[16] R. D. Martin, S. Rachev, and F. Siboulet, "Phi-alpha optimal portfolios and extreme risk management," Wilmott Magazine of Finance, vol. 2003, no. 6, pp. 70-83, 2003.

[17] V. Chow and C. W. Lai, "Conditional sharpe ratios," Finance Research Letters, vol. 12, pp. 117-133, 2015.

[18] L. Chen, S. He, and S. Zhang, "When all risk-adjusted performance measures are the same: in praise of the sharpe ratio," Quantitative Finance, vol. 11, no. 10, pp. 1439-1447, 2011.

[19] Y. Liu, X. Wu, and F. Hao, "A new chance-variance optimization criterion for portfolio selection in uncertain decision systems," Expert Systems with Applications, vol. 39, no. 7, pp. 6514-6526, 2012.

[20] X.-L. Wu and Y.-K. Liu, "Optimizing fuzzy portfolio selection problems by parametric quadratic programming," Fuzzy Optimization and Decision Making, vol. 11, no. 4, pp. 411-449, 2012.

[21] S. Wang and Y.-K. Liu, "Modeling renewal processes in fuzzy decision system," Applied Mathematical Modelling, vol. 39, no. 5-6, pp. 1536-1553, 2015.

[22] S. Wang, T. S. Ng, and M. Wong, "Expansion planning for wasteto-energy systems using waste forecast prediction sets," Naval Research Logistics, vol. 63, no. 1, pp. 47-70, 2016.

[23] Y. J. Chen, Y. K. Liu, and X. L. Wu, "A new risk criterion in fuzzy environment and its application," Applied Mathematical Modelling, vol. 36, no. 7, pp. 3007-3028, 2012.

[24] X. J. Bai and Y. K. Liu, "Semideviations of reduced fuzzy variables: a possibility approach," Fuzzy Optimization and Decision Making, vol. 13, no. 2, pp. 173-196, 2014.

[25] T. Hasuike and H. Katagiri, "Sensitivity analysis for random fuzzy portfolio selection model with investor's subjectivity," IAENG International Journal of Applied Mathematics, vol. 40, no. 3, pp. 185-189, 2010.

[26] R. Raei and M. B. Jahromi, "Portfolio optimization using a hybrid of fuzzy ANP, VIKOR and TOPSIS," Management Science Letters, vol. 2, no. 7, pp. 2473-2484, 2012.
[27] S. Wang and J. Watada, Fuzzy stochastic optimization: Theory, Models and Applications, Springer-Verlag, New York, NY, USA, 2012.

[28] T. T. Nguyen, L. Gordon-Brown, A. Khosravi, D. Creighton, and S. Nahavandi, "Fuzzy portfolio allocation models through a new risk measure and fuzzy sharpe ratio," IEEE Transactions on Fuzzy Systems, vol. 23, no. 3, pp. 656-676, 2015.

[29] S. Wang, J. Watada, and W. Pedrycz, "Granular robust mean$\mathrm{CVaR}$ feedstock flow planning for waste-to-energy systems under integrated uncertainty," IEEE Transactions on Cybernetics, vol. 44, no. 10, pp. 1846-1857, 2014.

[30] S. Wang and W. Pedrycz, "Robust granular optimization: a structured approach for optimization under integrated uncertainty," IEEE Transactions on Fuzzy Systems, vol. 23, no. 5, pp. 1372-1386, 2015.

[31] Z.-Q. Liu and Y.-K. Liu, "Type-2 fuzzy variables and their arithmetic," Soft Computing, vol. 14, no. 7, pp. 729-747, 2010.

[32] Y. Liu and Y.-K. Liu, "The lambda selections of parametric interval-valued fuzzy variables and their numerical characteristics," Fuzzy Optimization and Decision Making, vol. 15, no. 3, pp. 255-279, 2016.

[33] M. Carter and B. van Brunt, The Lebesgue-Stieltjes Integral, Undergraduate Texts in Mathematics, Springer, Berlin, Germany, 2000.

[34] Y. Liu and Y.-K. Liu, "Distributionally robust fuzzy project portfolio optimization problem with interactive returns," Applied Soft Computing, 2016.

[35] B. Liu and Y.-K. Liu, "Expected value of fuzzy variable and fuzzy expected value models," IEEE Transactions on Fuzzy Systems, vol. 10, no. 4, pp. 445-450, 2002.

[36] A. Charnes and W. W. Cooper, "Programming with linear fractional functionals," Naval Research Logistics Quarterly, vol. 9, no. 3-4, pp. 181-186, 1962.

[37] H. P. Benson, "Fractional programming with convex quadratic forms and functions," European Journal of Operational Research, vol. 173, no. 2, pp. 351-369, 2006.

[38] Y.-K. Liu and J. Gao, "The independence of fuzzy variables with applications to fuzzy random optimization," International Journal of Uncertainty, Fuzziness and Knowledge-Based Systems, vol. 15, pp. 1-20, 2007. 


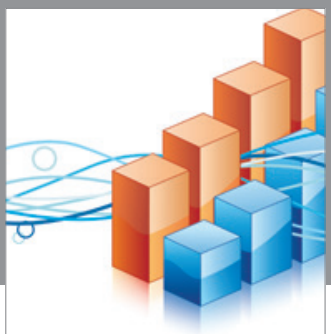

Advances in

Operations Research

vatem alat4

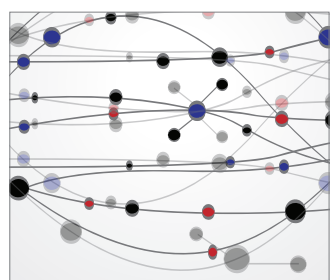

\section{The Scientific} World Journal
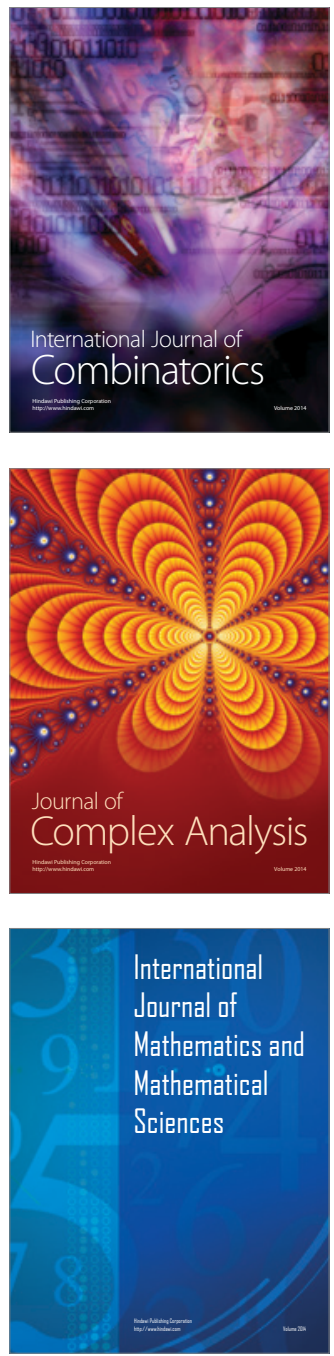
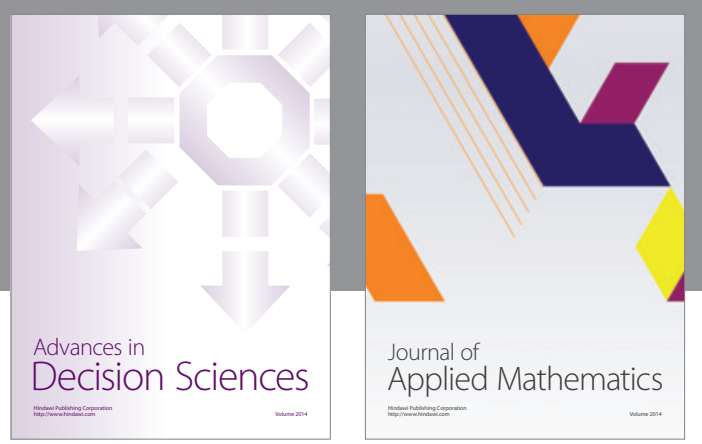

Algebra

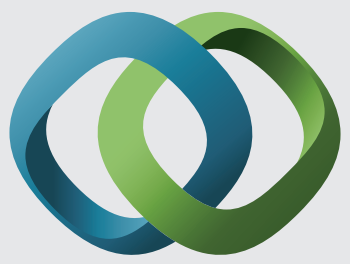

\section{Hindawi}

Submit your manuscripts at

https://www.hindawi.com
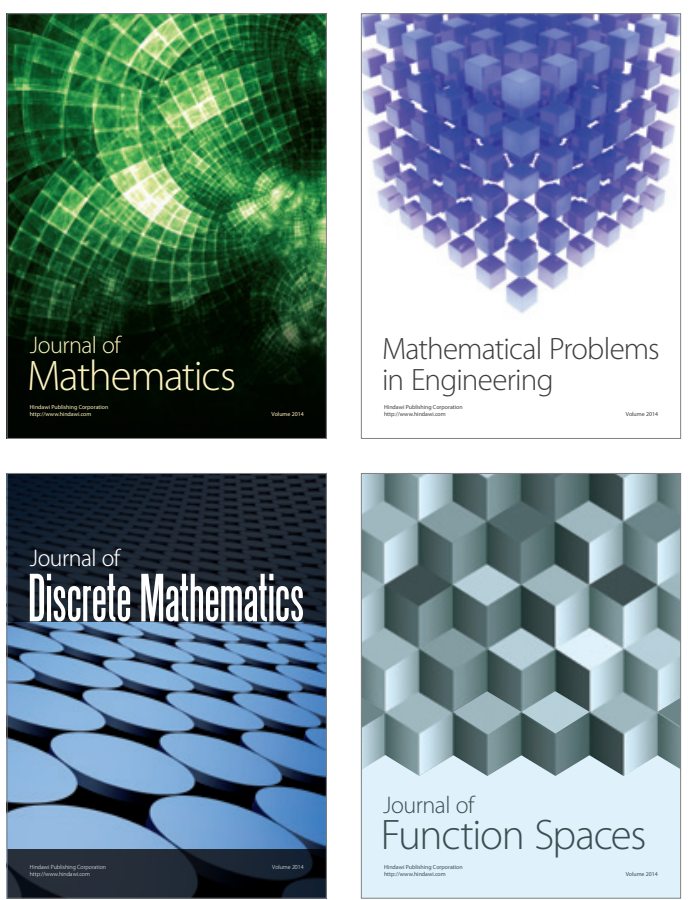

Mathematical Problems in Engineering
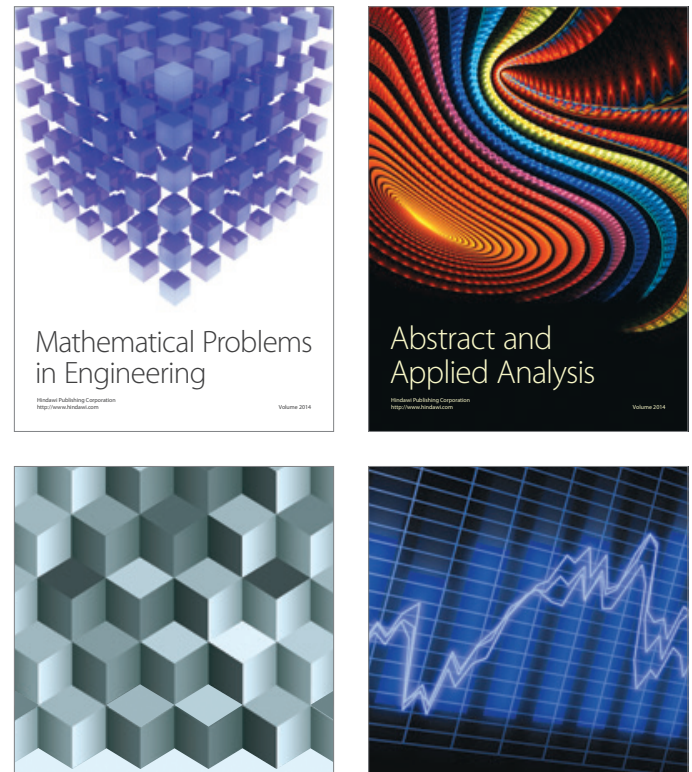

Journal of

Function Spaces

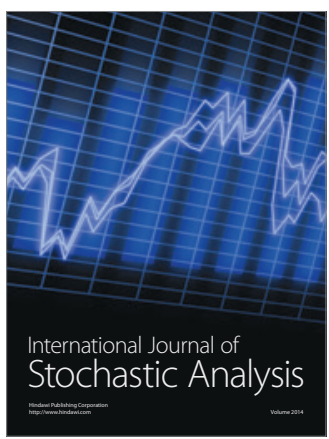

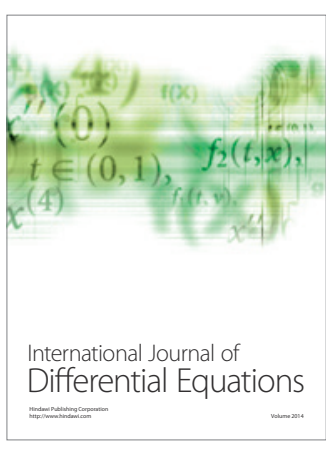
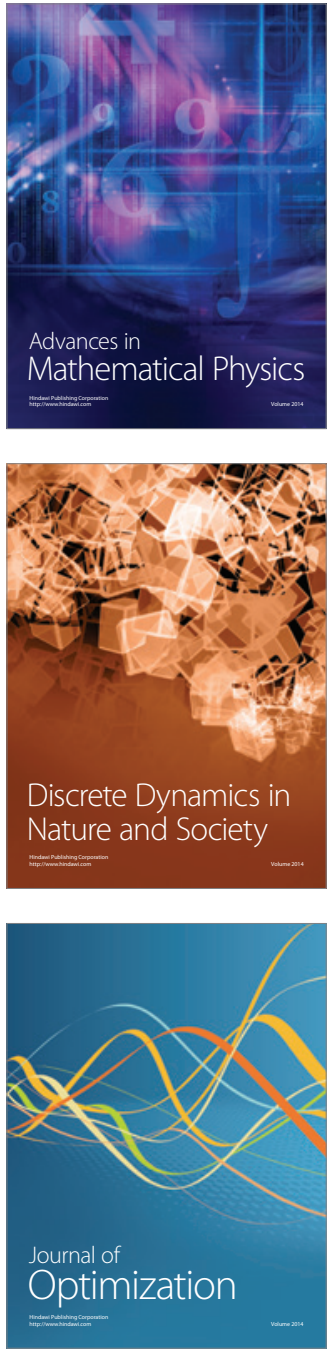